\title{
Commitment, Collaboration, and Problem Resolution to Promote and Sustain Access to Multifaceted Applied Behavior-Analytic Services Utilizing Telepractice
}

\author{
Janice K. Frederick ${ }^{1}$ - Valerie R. Rogers ${ }^{1}$ (D) - Ginger R. Raabe ${ }^{1}$ \\ Accepted: 21 December 2020 / Published online: 15 April 2021 \\ (C) Association for Behavior Analysis International 2021
}

\begin{abstract}
Access to clinically recommended applied behavior analysis (ABA) services has been significantly impacted for many consumers in light of the global COVID-19 pandemic. Local shelter-in-place orders and safety concerns have resulted in a movement toward telepractice models across educational and medically necessary ABA services, including at the level of the behavior technician. With this novel mode of technician-level intervention, practitioners have faced many learner, caregiver, and setting variables that have served as barriers to accessing telepractice intervention. Given the novelty of and limited empirical investigations on technician-level telepractice, solutions for common barriers are urgently needed. The current discussion article, therefore, describes the necessity of evaluating the efficacy of telepractice at the level of the technician, puts forth the position that telepractice should be considered a safeguard to accessing ABA intervention given evolving crises such as the pandemic, and provides a detailed description of employed training models and materials, problem-resolution strategies aimed at overcoming specific barriers, and initial outcomes across educational and medically necessary intervention models with the intent to support practitioners in identifying and overcoming barriers such that consumers can access needed intervention.
\end{abstract}

Keywords Applied behavior analysis $\cdot$ COVID-19 $\cdot$ Telepractice $\cdot$ Telehealth $\cdot$ Problem resolution $\cdot$ Treatment access

The 2019 coronavirus (COVID-19) pandemic continues to rage with no clear indication of when or how it will come to an end. Epidemiologists across the world have set forth projections in an effort to mitigate the impact of COVID-19, and although their forecasts of the virus's trajectory vary, they all agree that the future depends on a number of unknowns (Scudellari, 2020). In the United States, there have been great variability and division across and within states related to the reopening of businesses and schools, as well as the criteria for reinitiating shutdowns and other restrictions. With respect to schools, many campuses remain closed, and students are receiving instruction remotely either in entirety or under a hybrid model. Reports indicate that more than $84 \%$ of students

Janice K. Frederick

janice.frederick@abrite.org

1 The ABRITE Organization, 749 37th Ave, Santa Cruz, CA 95062, USA nationwide are participating in some level of remote instruction (Education Week, https://www.edweek.org/ew/section/ multimedia/school-districts-reopening-plans-a-snapshot. $\mathrm{html}$ ).

Not only has the pandemic resulted in tremendous disruption to educational systems, but the impact reaches well beyond public and private schools serving the $\mathrm{K}-12$ population (United Nations, 2020). Access to early learning programs (e.g., preschools), as well as support and intervention services (e.g., Head Start, early intervention programs) and various therapeutic and social services previously provided in person, is now greatly reduced. Some of these programs have been closed, whereas others have attempted transitions to remote service models with varying degrees of success. Applied behavior analysis (ABA) intervention is no exception. Temporary closures of schools and center-based ABA facilities, agency shutdowns, staffing limitations, and exposure-related complications are some of the many potential threats to access to services for many learners. In summary, given the current pandemic, multiple barriers have obstructed and may continue to obstruct learner access to in-person 
educational, social, and medically necessary services for the foreseeable future.

\section{Concurrent Implementation and Empirical Evaluation Due to Crisis}

Remote service delivery is being used across sectors to alleviate the extent to which access to these services is impacted; however, the empirical literature related to the provision and efficacy of some of these services, including ABA, is relatively limited. Remote service delivery, often labeled as remote instruction, distance learning, or telehealth, is hereafter referred to as telepractice to encompass both medically necessary and educational services. For the purposes of this article, telepractice involves live audio and/or videoconferencing between a learner and support staff. In recent years, research examining $\mathrm{ABA}$ services delivered via telepractice has expanded and yielded promising results related to outcomes for treating undesirable behavior (Romani \& Schieltz, 2017), as well as teaching functional replacement skills (Lindgren et al., 2016; Simacek, Dimian, \& McComas, 2017). A great deal of the research has focused on telepractice delivered by a Board Certified Behavior Analyst (BCBA) or an individual with advanced graduate-level training in behavior analysis wherein the behavior analyst works more directly with the client's caregiver via a consultative or coaching model (Lindgren et al., 2016; Schieltz \& Wacker, 2020; Wacker et al., 2013). ABA intervention is often delivered under a tiered service delivery model with direct intervention provided by an individual with training in behavior-analytic techniques, such as a behavior technician (BT), under the direction of a trained behavior analyst. The level of training and credentialing varies across models. Empirical literature documenting ABA telepractice delivered by a BT as a direct service to the learner is limited. In the recent emergency publication series in Behavior Analysis in Practice, some authors outlined guidance and provided decision-making tools related to direct telehealth at the BT level (Colombo, Wallace, \& Taylor, 2020; Frederick, Raabe, Rogers, \& Pizzica, 2020; Rodriguez, 2020); however, data are still needed to examine the efficacy of these recommendations.

Despite the need for more data-based analysis of these frameworks, given the impacts of the current pandemic, practioners are not in a position to wait until the research data are published to move forward with the implementation of BT telepractice, as the bulk of ABA service hours tends to be authorized at the level of the BT. For example, authorizations in alignment with the literature on optimizing outcomes for learners with autism may be prescribed at the rate of as many as $40 \mathrm{hr}$ a week (Healy \& Sinéad, 2013). Under conditions impacting in-person service delivery, access to these hours may be restricted to remote delivery. It is critical that behavior analysts use clinically sound procedures informed by the empirical literature and engage in continuous measurement, analysis, and revision of practices and models of service delivery at the individual-learner level and across learners. These practices should occur across all conditions of intervention (e.g., during distance-learning conditions and medically necessary services), given that learner needs and the roles of the BT may differ across conditions. Implementation and analysis can occur concurrently through the use of a scientist-practitioner approach (Hayes, Barlow, \& Nelson-Gray, 1999) that recognizes the urgency of such work, given the lack of existing research and the threats to accessing services imposed by the pandemic. It is only through the description and dissemination of procedures and associated data, including those that yield positive and less successful outcomes, that a cohesive set of practices guideing all components of ABA telepractice can be identified.

\section{Telepractice as a Safeguard for Access}

Frameworks examining the viability of ABA telepractice as an option for direct treatment may assume a degree of choice or view it as a possible alternative to in-person intervention. In particular sectors, such as publicly funded education, telepractice may be the only option for receipt of BT supports because of county-specific mandates and restrictions. There are other situations wherein telepractice may be the only option to avoid the complete interruption of ABA services or to allow the learner to access the rate of ABA treatment mandated by a service authorization. For example, telepractice may be the only option in the context of confirmed or potential COVID-19 exposures, learners or their family members who are at high risk, parent/caregiver refusal of in-person service, staffing limitations, and a lack of an appropriate setting for the continuation of in-person intervention. Moreover, these contexts could develop with little or no warning, requiring a swift transition from in-person to telepractice services to avoid disruptions to services and progress. For this reason, it may be beneficial to engage in preparatory activities related to telepractice with all learners and their caregivers, including those presently receiving and providing in-person services.

The need for alternatives to in-person ABA intervention is clear given the prevalence of variables that may threaten its sustainability at recommended levels. Yet novel applications of ABA technologies - as well as the related development of the training, processes, supports, and evaluative analyses that these applications require - may be insuperable in the time of a pandemic. Our organization supports learners via multiple service lines, including medically necessary programs and special education programs based in nonpublic agencies (NPAs), nonpublic schools (NPSs), and collaborative school districts. When school campuses were closed in the spring of 
2020 , all of our learners supported through school district funding had a sudden disruption and loss of services. This unforeseen transition required an immediate shift in service delivery and the recognition that learners' access to intervention in all service lines was at risk.

In an effort to mitigate service loss and driven by a value to sustain access to critical supports for learners, we developed models and tools to implement telepractice across levels (e.g., BT, BCBA) and lines of service delivery. Frederick et al. (2020) provided a model and related reference materials for BT and BCBA telepractice support services in the context of remote special education programs. This model of telepractice for special education students was developed, funded, and implemented under an abbreviated timeline. The collaboration and advocacy that led to its funding were driven in many ways by a sense of complete desperation imposed by a threat to the value of sustaining learners' services. This early learning was then used to implement BT telepractice for learners receiving insurance-funded, medically necessary services.

When approval for BT telepractice via insurance funding was secured, our organization initially considered its initiation for only those learners for whom in-person treatment was not a possibility or was impacted by a staffing need. This changed as our data set grew, concurrent with increasing restrictions and threats to accessing in-person treatment. Thus, we shifted to a position that telepractice should be immediately considered for all learners served by our organization. Our position evolved from using telepractice only by necessity to exploring it with all learners as a means to increase access to service and/ or in preparation for a sudden change in access. This position, and the commitment to it by our clinical and administrative team, required additional time allocation and financial resources for the development of tools, training, and evaluation of telepractice. It also moved the team from a place of uncertainty related to BT telepractice and a wavering with respect to implementation to a place of systematic consideration and universal learner assessment related to fitness for telepractice.

These models were not implemented without obstacles, but through the use of a scientist-practitioner approach, the components of the model and related tools continued to become more comprehensive, effective, and useful across learner profiles and placements. The methods and materials shared here must be subjected to additional analyses before more definitive conclusions can be drawn related to their effectiveness. However, the sharing of these data on telepractice and related outcomes, including social validity measures, has already resulted in increased access to ABA intervention for many learners within our organization. For example, there was a $40 \%$ increase in daily hours (from 3 to 5 or more hr per school day) of support for NPA students participating in our distancelearning programs. In addition, within the initial 1-month period following shelter-in-place (SIP) orders in California, our BT telepractice hours increased from 0 to more than $1,600 \mathrm{hr}$, with subsequent increases to $3,000 \mathrm{hr}$ of BT telepractice delivered in the 2nd month of the SIP order. Learners across a range of ages, skill levels, behavioral excesses, diagnoses, and other demographics have been served by our organization via direct telepractice at both the BCBA and BT support levels. Across service lines, over 12,000 hr of BT-level intervention were delivered to learners who might not have had access to services in the absence of the aforementioned telepractice models.

It is the hope that sharing these training models and materials, outcomes, and strategies for problem resolution will assist school districts, ABA agencies, and individual ABA service providers in facilitating access to intervention for learners. This goal is particularly relevant under conditions wherein telepractice may be the only option. This dissemination is in alignment with our value to promote and sustain access to ABA intervention for learners and their families. This value is always primary in decision making for our organization, but intensely so during this time when it is threatened by a crisis with such an unpredictable path. Our goal is to disseminate our approach and materials based on promising preliminary outcomes for our learners, as well as for our internal and external teams. We have attempted to include as many details as possible so that others will be aware of logistical variables (e.g., increased training costs) and potential applications of the methodologies and materials shared. We also hope that sharing this information will encourage structured, evaluative analyses of similar approaches. Specifically, this article will describe (a) training and supports provided to BTs and BCBAs related to telepractice, (b) collaboration with parents and funding sources, (c) learner assessment and prerequisite skill development, (d) learner profiles and problemresolution strategies, and (e) preliminary learner outcomes and social validity measures.

\section{Foundations of Implementation of BT Telepractice}

\section{Training and Support of the Internal Team}

The implementation of direct telepractice by BTs, as well as supervising clinicians, involved commitment and investment in the training of our team on both the technology and the clinical components of service delivery. All clinical team members were provided with training in preparation to engage in telepractice, including workstation and equipment setup and materials requirements (Appendix A). A visual check was conducted via video or photo to make certain that initial preparatory requirements were met and that setting consistency could be verified. All clinical team members participated in training related to technology, including using hardware, initiating sessions, and troubleshooting. Each team member was 
required to demonstrate competency in these skills via a direct assessment prior to receiving training in the clinical components of telepractice.

BT Training on Direct Telepractice As mentioned previously, our organization supports learners in the context of both educational and medically necessary programs. The role of the BT varies across and within these programs depending on individualized learner needs and goals. All BTs participated in an initial training sequence related to direct telepractice. They were then provided with additional training specific to the contexts in which they provided support. The initial telepractice clinical training sequence was related to professionalism and ethics, rapport building, prompting, session structure, antecedent behavior management strategies, and reinforcer identification and delivery (Table 1). In most cases, BTs were provided with supervisor-level overlap supports during their initial one to two telepractice sessions. During these sessions, in vivo modeling and the chat function were used to provide immediate feedback to the BT. Delayed feedback was provided via a telepractice-specific performance checklist.

Group-based training for BTs was increased from once monthly to weekly or biweekly, depending on the BT's service-delivery context to address telepractice clinical skill development and competencies. The initial BT telepractice training sequence was approximately $3 \mathrm{hr}$. Subsequent ongoing group-based training increased from $2 \mathrm{hr}$ monthly to approximately $4 \mathrm{hr}$ during the initial 3 months of our organization's implementation of direct telepractice. Eighty-five BTs were trained to deliver telepractice in the initial month of BT telepractice service delivery. Over 150 BTs have now participated in initial and ongoing training in telepractice. A survey was conducted with the BTs who participated in telepractice training to learn which content area was regarded as most valuable and areas in which additional training would be helpful. This survey was not anonymous and was administered electronically through a survey platform. An analysis of our survey results revealed that learning about session structure was the most valuable aspect of BTs' telepractice training (36\% of respondents), whereas the responses to the other training options were evenly distributed across building rapport, delivering reinforcers, and technology. In addition, the majority of the participants $(61 \%)$ reported that follow-up training on treating challenging behavior would be helpful, with a lower percentage of participants $(23 \%)$ requesting more training regarding the role of parents in supporting telepractice sessions.

Training for the BCBAs BCBAs were provided with training related to technology and ethical considerations, as well as an orientation on the empirical literature demonstrating the efficacy of parent training, consultation, and supervision by a
BCBA via telepractice (e.g., Boisvert, Lang, Andrianopoulos, \& Boscardin, 2010; Ferguson, Craig, \& Dounavi, 2019; Tomlinson, Gore, \& McGill, 2018). Training for the BCBAs subsequently focused on learner assessment, programming, supervision related to BT direct telepractice, coordination with caregivers, and problem resolution related to service delivery. BCBAs were trained in, and asked to follow, a specific set of sequential activities as they examined the initiation of BT-level telepractice for each of their learners (Fig. 1, Telepractice Barriers Assessment). These steps included (a) obtaining consent, (b) conducting a learner assessment, (c) engaging in caregiver assessment and collaboration, (d) carrying out telepractice probe sessions, (e) developing individualized learner recommendations and supports, and (f) planning for increased session observations and feedback. These steps were similar to those outlined in the Practice Parameters for Telehealth-Implementation for Applied Behavior Analysis set forth by the Council of Autism Service Providers (n.d.). Training was delivered to BCBAs in a variety of formats, including live and videobased instruction and handouts (Table 2).

A focal point of training for BCBAs involved providing support for and engaging in collaborative treatment planning with caregivers during this period fraught with additional stressors. More specifically, BCBAs received training in how to serve as an empathic listener and to seek input from caregivers about the evolving needs of their child and family during this time of crisis. BCBAs also participated in discussion and role-play scenarios related to working with caregivers to examine any proposed or predicted changes that may impact learners' access to intervention, such as how, when, and where services would be delivered. This also included exploring the viability of BT-level telepractice with caregivers to increase and sustain learners' access to intervention. BCBAs were also provided with models of individualized learner recommendations and supports, including session structures and reinforcement and behavior reduction protocols modified for implementation via telepractice.

In addition to process-related training and support materials, BCBAs participated in training related to problem resolution on the initiation and ongoing implementation of BT telepractice based on learner profiles found to be prevalent during the initial phase of BT telepractice implementation. Although these profiles are described in greater detail later (see the Implementation of BT Telepractice and Problem Resolution Model section), the training involved large and smaller BCBA groups working collaboratively to review individual parent and/or learner data, discuss and plan for the implementation of protocols designed to address telepractice-related barriers that challenged learners' access to intervention, and develop additional tools to support problem resolution.

Group-based training for BCBAs was increased considerably to allow for needed levels of support and opportunities to 
Table 1 Overview of training elements for BT-level practitioners

\begin{tabular}{|c|c|c|}
\hline Training topic & Concepts & Training activities \\
\hline \multirow[t]{4}{*}{$\begin{array}{l}\text { Professionalism } \\
\text { and ethics }\end{array}$} & \multirow[t]{2}{*}{$\begin{array}{l}\text { BACB RBT Ethics Code, including parent interactions } \\
\text { within telehealth }\end{array}$} & $\begin{array}{l}\text { Review and model various scenarios, compiled after BCBA-led } \\
\text { online probes. }\end{array}$ \\
\hline & & $\begin{array}{l}\text { Role-play and hold Q\&A with staff regarding reviewed } \\
\text { telehealth scenarios and appropriate parent interactions, } \\
\text { from needing clinical support from parents, to the delivery of } \\
\text { reinforcers or support with behavior management. }\end{array}$ \\
\hline & \multirow[t]{2}{*}{ Telehealth workstation requirements } & $\begin{array}{l}\text { Review appropriate workstation standards, including limiting } \\
\text { distractions, a professional background, professional attire, } \\
\text { and space to conduct sessions, as well as the presentation of } \\
\text { stimuli, various toys as needed, and learner materials. }\end{array}$ \\
\hline & & $\begin{array}{l}\text { Review Zoom troubleshooting generally, including resolving } \\
\text { connectivity issues, creating meetings, and using features } \\
\text { such as screen and content sharing. }\end{array}$ \\
\hline \multirow[t]{2}{*}{ Building rapport } & \multirow[t]{2}{*}{$\begin{array}{l}\text { Applying trained rapport-building techniques to telehealth, } \\
\text { including providing noncontingent attention, reducing demands, } \\
\text { and pairing with established reinforcers in the virtual environment }\end{array}$} & $\begin{array}{l}\text { Present scenarios and have staff provide examples as } \\
\text { to how to establish rapport in these contexts with } \\
\text { hypothetical learners and interests. }\end{array}$ \\
\hline & & $\begin{array}{l}\text { Review Zoom capabilities with regard to using features that } \\
\text { promote learner engagement and attending (e.g., changing } \\
\text { your background to a preferred picture, using electronic } \\
\text { token boards with "stickers" of preferred characters). }\end{array}$ \\
\hline \multirow[t]{2}{*}{ Prompting } & $\begin{array}{l}\text { Review of existing prompts and modifications to hierarchies, } \\
\text { namely physical prompts }\end{array}$ & $\begin{array}{l}\text { Share and review telehealth prompt-hierarchy document with } \\
\text { staff. Review sample programs and how they could be } \\
\text { modified in the absence of physical support. Review sample } \\
\text { teaching instructions that BCBAs may provide. }\end{array}$ \\
\hline & $\begin{array}{l}\text { Instruction on enlisting parent support in case physical guidance } \\
\text { is needed }\end{array}$ & $\begin{array}{l}\text { Review options to communicate with parents (via email when } \\
\text { sending the Zoom link, or using the chat feature) so parents } \\
\text { can be instructed and supported. }\end{array}$ \\
\hline \multirow[t]{2}{*}{ Session structure } & $\begin{array}{l}\text { Structuring time in telehealth sessions with increased rapport } \\
\text { building }\end{array}$ & $\begin{array}{l}\text { Provide sample session structures, looking at increased rapport } \\
\text { building and highlighting the skills that are most functional } \\
\text { and applicable for the learner, especially in initial telehealth } \\
\text { sessions. }\end{array}$ \\
\hline & How and when to target skill acquisition & $\begin{array}{l}\text { Discuss the potential for increased intertrial intervals or pacing } \\
\text { between programs to allow for increased reinforcement for } \\
\text { attending and engagement. }\end{array}$ \\
\hline \multirow[t]{2}{*}{$\begin{array}{l}\text { Behavior } \\
\text { management }\end{array}$} & $\begin{array}{l}\text { Emphasis on existing or increased antecedent } \\
\text { strategies based on topography and function }\end{array}$ & $\begin{array}{l}\text { Review sample behavior protocols; this training builds upon } \\
\text { rapport building and session structure to ensure preemptive } \\
\text { environmental arrangements can be made to reduce the } \\
\text { likelihood of maladaptive behaviors. }\end{array}$ \\
\hline & $\begin{array}{l}\text { Modifications to consequence manipulations and the use } \\
\text { of parent support if needed due to topography or function }\end{array}$ & $\begin{array}{l}\text { Review sample behavior protocols with respect to managing } \\
\text { maladaptive behaviors at a distance, including reaching out to } \\
\text { parents in the case of elopement or a child intentionally logging } \\
\text { off the meeting. }\end{array}$ \\
\hline \multirow[t]{3}{*}{$\begin{array}{l}\text { Reinforcer } \\
\text { identification } \\
\text { and delivery }\end{array}$} & How to transition existing reinforcers to a telehealth model & $\begin{array}{l}\text { Discuss with staff about existing reinforcers and how they can } \\
\text { translate to telehealth, such as having physical toys and } \\
\text { showing them on camera to engage in imaginary play with } \\
\text { dolls or action figures. }\end{array}$ \\
\hline & \multirow[t]{2}{*}{$\begin{array}{l}\text { How to use aspects of online service delivery to expand } \\
\text { on reinforcer repertoire, including screen sharing, videos, } \\
\text { games, and features of Zoom }\end{array}$} & $\begin{array}{l}\text { Review the features of Zoom, including screen sharing. Share } \\
\text { and update ongoing tip sheets and reinforcer lists, including } \\
\text { links to games and videos. }\end{array}$ \\
\hline & & $\begin{array}{l}\text { Hold staff Q\&A, as well as discussions related to training } \\
\text { scenarios presented and creative ways to engage and present } \\
\text { reinforcers. Review varying social praise and give additional } \\
\text { examples. }\end{array}$ \\
\hline
\end{tabular}

Note. BACB RBT Behavior Analyst Certification Board Registered Behavior Technician, $B T$ behavior technician, $B C B A$ Board Certified Behavior Analyst.

share and review data as a team. More specifically, organization-wide BCBA training was increased from $3 \mathrm{hr}$ monthly to approximately $3 \mathrm{hr}$ nearly weekly throughout the initial 3 months of telepractice implementation. Results from surveys conducted with BCBAs revealed that the majority of our BCBA-level team found our group-based trainings to be the most helpful. BCBAs reported that they found the description of specific learner challenges and the sharing of their assessment and telepractice intervention data to be extremely helpful as a training mechanism. 

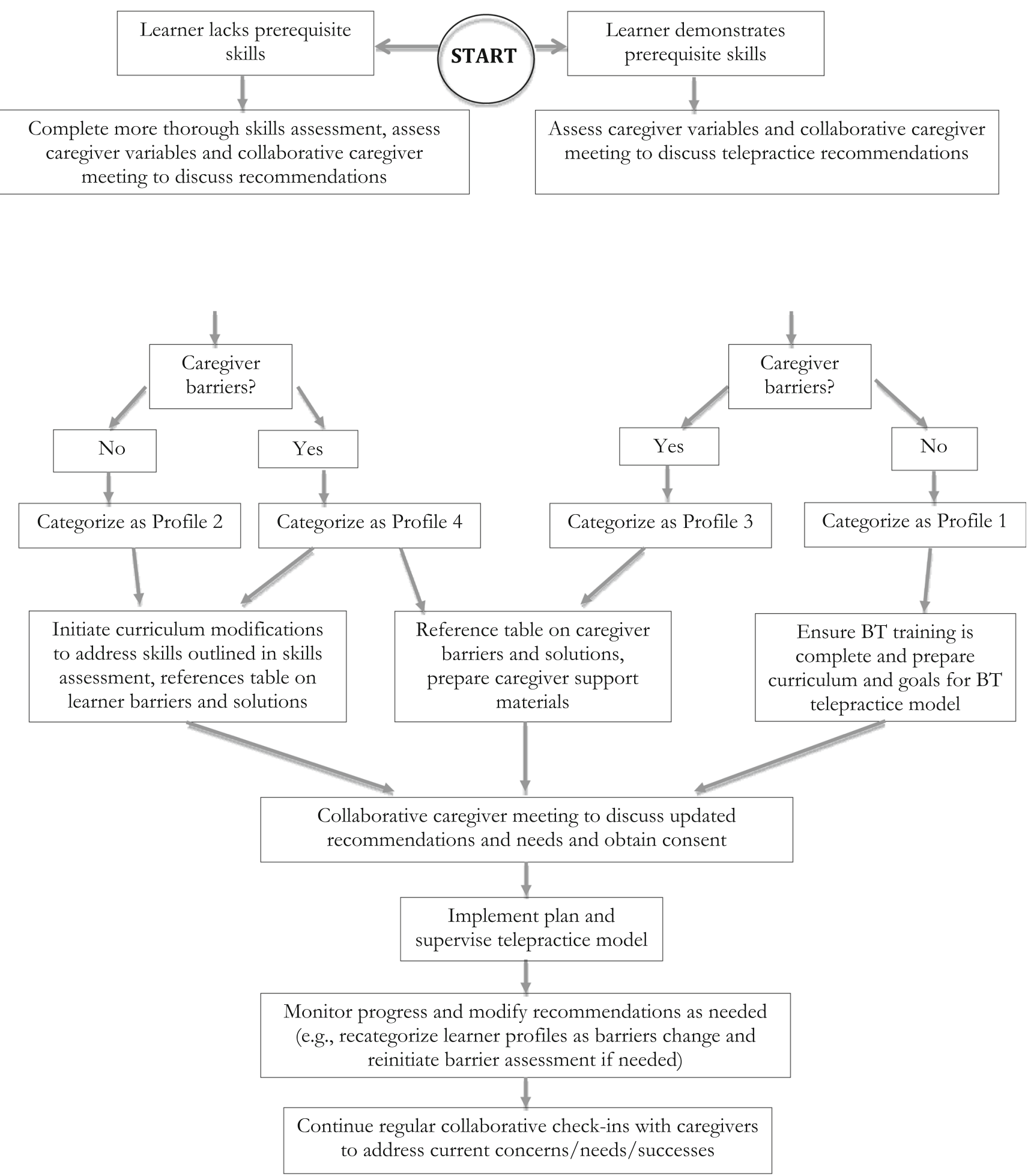

Fig. 1 Telepractice barriers assessment. Note. $\mathrm{BCBA}=$ Board Certified Behavior Analyst; $\mathrm{BT}=$ behavior technician

\section{Collaboration With Parents and External Teams}

A critical component of the efforts to sustain our learners' access to intervention involved collaboration with parents and external teams, including local and county-level school district administrators. The absence of collaboration with parents and our external teams would have significantly reduced, if not terminated, access to intervention for a majority of our learners during the pandemic. For example, countylevel restrictions on service delivery for those receiving support via school district funding meant that telepractice was the only option to sustain intervention. For all of our learners, 
Table 2 Overview of training elements for the BCBA-level practitioners

\begin{tabular}{|c|c|c|}
\hline Training topic & Concepts & Training activities \\
\hline $\begin{array}{l}\text { Orientation to empirical } \\
\text { literature }\end{array}$ & $\begin{array}{l}\text { Review of research examining } \\
\text { ABA and telepractice }\end{array}$ & $\begin{array}{l}\text { Summarize and discuss published studies examining } \\
\text { telepractice, their results, and gaps in the empirical literature. } \\
\text { Provide access to research articles, including those published } \\
\text { as a part of the emergency COVID-19 publication series } \\
\text { in Behavior Analysis in Practice. }\end{array}$ \\
\hline \multirow[t]{5}{*}{ Learner assessment } & $\begin{array}{l}\text { Methods for examining learner } \\
\text { fitness for telepractice }\end{array}$ & $\begin{array}{l}\text { Introduce and review the telepractice decision-making } \\
\text { flowchart (see Figure 1). }\end{array}$ \\
\hline & $\begin{array}{l}\text { Initial coordination with caregiver/external } \\
\text { teams }\end{array}$ & $\begin{array}{l}\text { Provide templates for questions designed to support learning } \\
\text { about the caregiver's/team's pandemic-related challenges } \\
\text { and concerns. }\end{array}$ \\
\hline & $\begin{array}{l}\text { Analysis of learner treatment plan, } \\
\text { caregiver and BCBA concern, and } \\
\text { potential impact of access }\end{array}$ & $\begin{array}{l}\text { Review the process for reviewing the learner's treatment plan } \\
\text { and outcomes in the context of current levels of access. }\end{array}$ \\
\hline & & $\begin{array}{l}\text { Highlight and model the steps involved in this analysis using } \\
\text { example learners. }\end{array}$ \\
\hline & $\begin{array}{l}\text { Prerequisite telepractice skill } \\
\text { assessment and identification of deficits }\end{array}$ & $\begin{array}{l}\text { Review the Telepractice Skills Assessment (see Appendix B) and inter- } \\
\text { pret results. }\end{array}$ \\
\hline \multirow[t]{2}{*}{ Programming for telepractice } & Adjustments to treatment protocols & $\begin{array}{l}\text { Review needed adjustments to session structures, reinforcement, } \\
\text { and behavior protocols. } \\
\text { Provide sample session structures and behavior protocols. } \\
\text { Provide a catalog of potential virtual stimuli, reinforcers, } \\
\text { and token boards, and demonstrate their use. }\end{array}$ \\
\hline & Telepractice probe sessions & $\begin{array}{l}\text { Provide an outline for BCBA telepractice probe sessions. } \\
\text { Provide samples of recorded, successful BCBA probe } \\
\text { telepractice sessions. }\end{array}$ \\
\hline \multirow{2}{*}{$\begin{array}{l}\text { Coordination with } \\
\text { BT-level practitioners }\end{array}$} & BT expectations for telepractice & Review the BT telehealth performance checklist. \\
\hline & BT support and training & $\begin{array}{l}\text { Provide review, group-based practice, and handouts related } \\
\text { to methods for supporting staff in vivo (e.g., private } \\
\text { chat feature in Zoom for immediate feedback). }\end{array}$ \\
\hline \multirow[t]{2}{*}{ Coordination with caregivers } & Steps to collaborative planning & $\begin{array}{l}\text { Hold direct and video-based training related to engaging } \\
\text { in collaborative planning with caregivers and external } \\
\text { teams. Role-play collaborative planning discussions } \\
\text { and review scenarios. }\end{array}$ \\
\hline & Compassionate care under crisis conditions & $\begin{array}{l}\text { Review guidelines for serving as an empathic and active } \\
\text { listener. Conduct role-play related to seeking } \\
\text { input. Discuss points and role-play related to working } \\
\text { with the caregiver to explore current and potential } \\
\text { impacts on the learner's access to services. }\end{array}$ \\
\hline \multirow[t]{2}{*}{ Problem resolution } & $\begin{array}{l}\text { Objective problem identification and } \\
\text { description to develop action plans }\end{array}$ & $\begin{array}{l}\text { Discuss extending current objective problem-solving } \\
\text { training and outline steps for telepractice-specific situations. }\end{array}$ \\
\hline & $\begin{array}{l}\text { Learner, caregiver, and environmental } \\
\text { barriers that may impact BT telepractice }\end{array}$ & $\begin{array}{l}\text { Review learner profile categorizations. Hold breakout } \\
\text { groups to identify barriers within each profile. Review } \\
\text { learner data related to utilized strategies, and share successes } \\
\text { and failures in addressing barriers. }\end{array}$ \\
\hline
\end{tabular}

Note. $B C B A$ Board Certified Behavior Analyst, $A B A$ applied behavior analysis, $B T$ behavior technician

sustaining access involved the exploration of telepractice with parents and external team members.

We recognized that, whereas a critical value driving our actions was continued access to intervention for our learners, this value and its level of prioritization might not align with those of each parent or external team member, given the multitude of pandemic-related issues that each individual, family unit, and external team could be facing. Therefore, we engaged in actions related to learning more about the needs of parents and external teams by using open-ended interviews, maintaining roles as active listeners related to interests and challenges, and working together to build models and formulate individual learner plans to introduce and/or establish preparedness for telepractice, given the threats to the continuation 
of in-person service delivery. Table 3 outlines guidance provided to BCBAs related to collaborative planning. As recommended by LeBlanc, Lazo-Pearson, Pollard, and Unumb (2020), we solicited repeated input from caregivers and team members and engaged in direct discussion related to their interests, including the introduction of telepractice. In some cases, we found that caregivers initially showed resistance to the introduction of telepractice even when it may have resulted in sustained access to services, but description and collaborative planning as a team led to its acceptance and introduction, which ultimately increased their access to service for many learners.

Steps taken to implement a distance support model for special education students served by our organization have been described previously (see Frederick et al., 2020), but it is worth mentioning that our advocacy related to access to services continued throughout implementation. Our methods have been further shaped by our learners, their caregivers, and external team members. The forms of advocacy that have yielded the most positive impact involved sharing individual learner stories and data, disseminating social validity data and collapsed learner outcomes, and regularly checking in and engaging with external team members. More specifically, our team regularly sent brief updates to school district administrators related to one to two student successes within the distance-learning model. These updates often included details related to the mastery of a specific skill or increases in engagement, a verbal report by the student related to the utility of the support, and/or positive feedback provided by a parent. Administrators relayed gratitude for these updates, indicating

Table 3 Steps to collaborative planning to support access to intervention

\begin{tabular}{lc}
\hline Step & Strategies/recommendations \\
\hline $\begin{array}{l}\text { The BCBA conducts a check-in with the caregiver/external team to ac- } \\
\text { knowledge the challenges regarding the pandemic and to learn more } \\
\text { about their variables and needs. }\end{array}$ & $\begin{array}{c}\text { The BCBA conducts the check-in via phone or videoconference to support } \\
\text { reciprocal engagement. }\end{array}$ \\
& $\begin{array}{c}\text { The BCBA uses open-ended interview/preplanned questions to support } \\
\text { BCBA ease with conducting check-in. }\end{array}$ \\
$\begin{array}{l}\text { The BCBA reviews the treatment plan, levels of service, and their concerns } \\
\text { related to the learner(s) should access be impacted, as well as the values, }\end{array}$ & $\begin{array}{c}\text { The BCBA asks themselves questions including "What are your concerns } \\
\text { about the learner(s)?" "What are the recent successes?" "What is needed } \\
\text { to support the current trajectory of treatment?" "Where is the }\end{array}$ \\
& $\begin{array}{l}\text { collaborative team trying to get to?" "What threats to access exist?" and } \\
\text { "What can mitigate these threats?" }\end{array}$
\end{tabular}

The BCBA conducts a follow-up meeting with the caregiver/external team and, if possible, with the learner(s) to discuss the intervention and the plan for any immediate or potential impacts to access.

The BCBA stays present and is an engaged listener during the meeting.

The BCBA shares values and efforts related to promoting access to intervention and encourages the caregiver/external team to outline their values. Both parties agree to open communication and the recognition that unknown conditions may shift values, needs, and access.

The BCBA and caregiver/external team make a commitment to open communication, collaboration, and preparedness for change and explore specific methods for sustaining and promoting access to intervention, including telepractice.

The BCBA shares information and experiences related to telepractice with the caregiver/external team.

The BCBA and caregiver/external team engage in collaborative problem resolution as needed.
The BCBA seeks input concerning preferences and concerns and shares their concerns and goals for the learner(s) in a direct manner.

The BCBA shares observations of the learner(s), including specific moments and outcomes that highlight their rapport and commitment.

The BCBA presents viable options to promote and sustain access to intervention, including telepractice, for learners.

The BCBA inquires regarding any barriers and takes notes regarding caregiver/external team notes related to telepractice assessment and/or initiation.

The BCBA remains in contact with the caregiver/external team at a regular frequency even if the intervention has been placed on hold.

The BCBA shares information related to successes and outcomes reported in the literature.

The BCBA shares information related to direct professional positive experiences.

The BCBA offers the caregiver/external team support via direct engagement and access to materials.

Note. More than one step may occur in the context of a single interaction. BCBA Board Certified Behavior Analyst. 
that they helped to show the positive impact that continued provision of services had on learners. They also reported that the updates were being shared with county superintendents and budgeting officers in an effort to sustain authorizations for ABA services. Data were shared with administrators in simple graphs with summary bullets to promote consumption and sharing among a wider range of external team members.

Our team also remained in close communication with school district and county administrators (engagement that was most often initiated by our team) to provide and receive information concerning potential impacts to service and to continue sharing preliminary outcome data. These engagements created collaboration opportunities related to the conceptualization of methods to remove any barriers to access faced by learners (e.g., internet instability at the learners' homes, provision of learning supplies, strategies to assess prerequisite skills for distance learning). Similarly, BCBAs shared successes from early telepractice sessions with caregivers using narratives supplemented with the learner's data.

In an effort to support the advocacy efforts by parents of learners whom we serve across programs, we appointed specific team members to maintain up-to-date information from funding sources, including insurers and educational agencies. This information was summarized and shared with caregivers in the context of training meetings, handouts, and videotaped segments provided via shareable links and posted on social media to increase accessibility (Table 4). These efforts were designed to promote caregiver awareness of state and federal guidance related to service access, to offer guidance related to sharing concerns with external teams (e.g., individualized education program team), and to provide strategies for effective sessions and self-care for learners and their caregivers.

\section{Implementation of BT Telepractice and Problem Resolution Model}

\section{Learner Assessment and Preparation}

After having collaborative discussions with parents and obtaining consent, we conducted assessments of telepractice readiness skills with all of our learners (see Appendix B for a specific list of telepractice readiness skills). As stated previously, for learners receiving ABA supports to access their educational programs, county restrictions resulted in telepractice being the only viable option for service delivery. For some learners attending our NPS or receiving medically necessary services through insurance funding, family health concerns (e.g., compromised immune systems or preexisting conditions) and caregiver preferences also resulted in an immediate need to assess and attempt the transition to telepractice. For the remaining learners for whom continuation of in-person intervention was possible, readiness was

Table 4 List of provided support materials

\begin{tabular}{|c|c|}
\hline Area of support & Support materials provided to caregivers \\
\hline $\begin{array}{l}\text { Technology and preparedness } \\
\text { of distance learning }\end{array}$ & $\begin{array}{l}\text { Training on technology and troubleshooting (direct training, handout) } \\
\text { Preparing for distance-education sessions (videotaped tips, handout, checklists) } \\
\text { Developing a workstation (videotaped tips, checklist) } \\
\text { Preparing for telepractice sessions (videotaped tips, handout, checklists) } \\
\text { Caregiver participation and support recommendations: group-based } \\
\quad \text { distance-learning sessions such as circle time and breakout groups } \\
\text { (videotaped tips) }\end{array}$ \\
\hline $\begin{array}{l}\text { Access to services with their } \\
\text { learner's school district }\end{array}$ & $\begin{array}{l}\text { Navigating the Department of Education's regulatory guidance related } \\
\text { to access to services (videotaped recommendations) } \\
\text { Communicating with their learner's IEP team regarding needs (videotaped tips, template) } \\
\text { Sharing learner and caregiver preferences and experiences with the IEP team } \\
\text { (videotaped tips, template) }\end{array}$ \\
\hline $\begin{array}{l}\text { Social-emotional aspects of } \\
\text { the COVID-19 pandemic }\end{array}$ & $\begin{array}{l}\text { Developing new routines (videotaped tips, handout, checklists) } \\
\text { Promoting connectedness with others when socially distanced (videotaped tips, } \\
\text { handout, activities for learners) } \\
\text { Identifying and exploring replacements for activities not available during the pandemic } \\
\text { (videotaped tips, activities for caregivers and learners) } \\
\text { Self-care and the importance of identifying values (videotaped tips) } \\
\text { Self-care exercises for learners and caregivers (interactive video module, exercises) }\end{array}$ \\
\hline
\end{tabular}

Note. IEP individualized education program. 
assessed as a preparatory measure should changing conditions require an immediate transition to telepractice.

Following the assessment of learner readiness, BCBAs completed a series of preparatory activities delineated by guidelines and an accompanying task checklist on which they received training. These activities included (a) conducting a probe telepractice session; (b) identifying goals to be targeted during BT telepractice sessions; (c) developing learner-specific telepractice session structures; (d) making needed modifications to prompting procedures, behavior intervention, and/or reinforcement protocols; (e) providing caregiver orientation and training related to technology and participation requirements; (f) preparing materials for instruction; and (g) conducting any necessary learner-specific training with the BT.

\section{Implementation and Identified Barriers}

Overall, the implementation of telepractice across learners and funding sources using the models developed was successful and moved in a relatively uniform manner, despite the variations in specific BT roles and conditions of service delivery. There were many learners who demonstrated prerequisite skills for telepractice and whose caregivers were supportive of initiation. For these learners, categorized as learners with prerequisite skills and no caregiver/setting barriers, the implementation of BT telepractice swiftly resulted in sustained or increased access to ABA intervention.

For a smaller subset of learners, obstacles were encountered, and as BT telepractice was initiated with an increasing number of learners, particular variables emerge that appeared to function as barriers to access. Some of the barriers to access that were related to learner variables included those described by Rodriguez (2020), such as poor attending skills and challenging behaviors. However, it was most often the case that the intersection of learner and caregiver/environmental variables that obstructed access was where the BCBAs reported being "stuck" or unable to readily reach resolution.

In an effort to identify strategies and develop tools to support the BCBAs in alleviating barriers to access, we initiated categorization of what we labeled "learner profiles," data collection and measurement of protocols implemented to support access, and analysis of protocols that generated increased access. The learner profiles used for categorization were (a) learner with prerequisite skills and no caregiver/setting barriers, (b) learner without prerequisite skills and no caregiver/ setting barriers, (c) learner with prerequisite skills and with caregiver/setting barriers, and (d) learner without prerequisite skills and with caregiver/setting barriers. Additionally, strategies to address learner and caregiver barriers (see Tables 5 and 6 for a brief description) and descriptions and outcome data for learners who met each profile are shared in an effort to support other practitioners in addressing similar barriers to access.
Profile 1: Learner With Prerequisite Skills and No Caregiver/ Setting Barriers Learners who met this profile demonstrated independence with prerequisite skills outlined in the telepractice readiness assessment. Collaborative discussion with the caregivers suggested that they were available and willing to facilitate during sessions as needed. This report was supported by data collected during telepractice probe sessions that suggested no caregiver or setting barriers to implementation. For these learners, little modification to intervention programs was needed to initiate BT telepractice.

Case Study Pat was an 8-year-old boy with a diagnosis of autism spectrum disorder (ASD) receiving $3.5 \mathrm{hr}$ per week of insurance-funded medically necessary treatment within his home prior to the SIP order. Pat's home included family members with compromised immune systems. Therefore, his family immediately placed in-person treatment sessions on hold, citing medical concerns. The BCBA supervising Pat's focused treatment program met with the family via a virtual platform to discuss the possible transition to telepractice services. The family shared that they were willing to attempt telepractice and were prepared to support Pat during treatment sessions as needed. With caregiver consent, the BCBA assessed Pat's readiness for telepractice sessions by completing the skills assessment checklist and then conducting a 1-hr probe telepractice session. Pat displayed all of the desired telepractice readiness skills, suggesting he was likely an appropriate candidate to receive telepractice services with limited modifications to his treatment plan, session structure, reinforcement systems, and behavior intervention plan. Telepractice treatment sessions at the BT level began with Pat receiving $4 \mathrm{hr}$ (two 2-hr sessions) weekly. After observing Pat's skill acquisition and reduction in challenging behaviors, as well as Pat's own report of his enjoyment of the telepractice sessions, his family agreed to increase his treatment hours to 8 $\mathrm{hr}$ weekly. With the transition to telepractice intervention and caregivers subsequently having more availability for intervention sessions, Pat received an additional $4.5 \mathrm{hr}$ of treatment weekly, when compared to the previous number of in-person sessions, and acquired skills at a faster rate (see Figure 2). Peer interaction and generalization goals were still able to be addressed via peer sessions on a virtual platform.

Profile 2: Learner Without Prerequisite Skills and No Caregiver/Setting Barriers Learners meeting this profile showed deficits in multiple prerequisite skills outlined in the telepractice readiness assessment, but caregiver collaboration and probes indicated that this barrier could be addressed through programming and caregiver facilitation and support. BCBAs described these learners as having the following difficulties: (a) attending to a screen, (b) sustaining a seated position for appropriate durations, (c) following directions and transitioning appropriately, and (d) having a limited number 
Table 5 List of examined caregiver barriers, potential solutions, and specific tips

Learner barrier Solution

Attending Shape attending to visual stimuli.

Shape attending to auditory stimuli.

Provide the caregiver with the protocol to target attending outside of the session.

Engagement

Following instructions

Teach following instructions delivered remotely.

Teach following instructions delivered at a distance.

Effective prompts

Have the caregiver deliver prompts in person.

Modify prompting hierarchy specific to the skill and telehealth.

Functional
reinforcers
reinforcers in person.
Establish conditioned reinforcers to be
delivered virtually.

Motor deficits Modify treatment plans/goals.

Have the caregiver prompt or facilitate actions requiring motor skills.

Have the caregiver support in communicating the learner's response.

Behavioral excesses Modify the behavior plan to address virtual instruction.

Increase antecedent manipulations and interventions.

Prioritize targeting replacement behaviors at an increased rate.

Train the caregiver to support/implement the behavior plan.
Tips

Target generalization of mastered skills to telehealth (using visual stimuli).

Use preferred stimuli, and teach visual tracking on screen.

Provide differential reinforcement for attending to the screen and green/camera light.

Shape increased duration of attending to visual stimuli.

Teach the learner to attend to instructions delivered at a distance, include responding to their name.

Teach the learner to attend to preferred auditory stimuli (e.g., songs).

Teach auditory discrimination skills without a visual component (e.g., learn to tact which item produced a given sound without seeing the item).

Use electronic-based games to track visual stimuli (e.g., pop balloons on screen).

Increase the use of video chat with family members.

Establish additional auditory and visual reinforcers.

Use interactive technologies and activities.

Provide differential reinforcement for proximity near the camera.

Use reinforcement systems for engagement in telehealth sessions.

Provide noncontingent breaks from the screen and physical movement, and systematically increase the duration of telehealth sessions.

Target skills via telehealth that were previously mastered in person.

Have the in-person caregiver deliver instructions at a distance prior to moving to telehealth-delivered instructions at a distance.

Have the in-person caregiver prompt following instructions delivered remotely.

Use rate-building/fluency procedures for mastered targets.

Train the caregiver on prompt delivery prior to use.

Remove physical prompting if the caregiver is unable to provide it.

Modify/add prompts (e.g., use of cursor as a gestural prompt).

Prioritize targets where verbal/visual prompts are most effective.

Train the caregiver on reinforcer delivery prior to use.

Create a list of potential electronic reinforcers for the BT to reference and use.

Have the caregiver restrict access to reinforcers needed for intervention.

Conduct a preference assessment of virtual reinforcers.

If motor deficit goals are prioritized, teach caregivers how to target them.

Train caregiver on prompting or completing motor tasks.

Use consequential modifications if needed given prompting/extinction constraints.

Use demand fading, noncontingent reinforcement, and differential reinforcement procedures.

Target mands for each function (specific mands may include mands for cessation, access to the caregiver, break from the screen, alternative location, etc.).

Specify the level of parent participation needed and conditions, and use behavioral skills training.

Note. BT behavior technician.

of effective reinforcers. Each of these barriers, as well as potential solutions and tips based on behavior-analytic principles and instructional strategies to address them, is outlined in Table 5. Some of the solutions used successfully by our team 
Table 6 List of examined caregiver barriers, potential solutions, and specific tips

\begin{tabular}{|c|c|c|}
\hline Caregiver barrier & Solution & Tips \\
\hline \multirow[t]{7}{*}{$\begin{array}{l}\text { Time/participation } \\
\text { Constraints }\end{array}$} & $\begin{array}{l}\text { Reexamine the schedule to see if revision } \\
\text { to the learner's availability is needed. }\end{array}$ & $\begin{array}{l}\text { Assist caregiver in determining changes to routine/schedule } \\
\text { to determine when sessions are possible, when caregivers } \\
\text { are home, etc. }\end{array}$ \\
\hline & Reduce the time required for the caregiver. & Consider modifying session duration or time (e.g., parent lunch hour). \\
\hline & Reduce response effort for the caregiver. & Isolate intervention goals that require little to no caregiver participation. \\
\hline & Involve siblings in sessions. & Target peer or generalization goals with siblings to reduce caregiver demands. \\
\hline & Specify caregiver responsibilities and needs. & Provide an outline of the caregiver's role and when support may be needed. \\
\hline & $\begin{array}{l}\text { Shape learner independence with session } \\
\text { engagement. }\end{array}$ & $\begin{array}{l}\text { Teach the learner how to initiate telehealth sessions (e.g., log } \\
\text { in to Zoom, troubleshoot tech problems, retrieve own materials). }\end{array}$ \\
\hline & & $\begin{array}{l}\text { Provide the learner with a checklist related to session components } \\
\text { and reinforce related responses. }\end{array}$ \\
\hline \multirow[t]{8}{*}{$\begin{array}{l}\text { Motivation (questions } \\
\text { efficacy) }\end{array}$} & $\begin{array}{l}\text { Detail and review data related to the learner's } \\
\text { prerequisite skills. }\end{array}$ & Share learner performance on the telepractice readiness assessment. \\
\hline & Demonstrate learner readiness and successes. & Supervisor to conduct a brief probe session with caregiver observation. \\
\hline & $\begin{array}{l}\text { Communicate the plan to ensure efficacy } \\
\text { specific to the learner. }\end{array}$ & Discuss recommended modifications to the intervention plan. \\
\hline & $\begin{array}{l}\text { Describe how telepractice may be different } \\
\text { from previous experiences. }\end{array}$ & $\begin{array}{l}\text { Identify differences between emergency distance learning } \\
\text { and planned telepractice. }\end{array}$ \\
\hline & $\begin{array}{l}\text { Discuss the clinical need for continued } \\
\text { access to intervention. }\end{array}$ & $\begin{array}{l}\text { Discuss the trajectory of learner progress based on learner } \\
\text { historical data. }\end{array}$ \\
\hline & $\begin{array}{l}\text { Provide access to additional information } \\
\text { and learner successes and outcomes. }\end{array}$ & $\begin{array}{l}\text { Shares learner successes and collapsed learner outcome data and information } \\
\text { from related articles. }\end{array}$ \\
\hline & & $\begin{array}{l}\text { Encourage the caregiver to follow telepractice-related posts } \\
\text { on social media. }\end{array}$ \\
\hline & & Send caregivers handouts/materials/links related to telehealth. \\
\hline \multirow{3}{*}{$\begin{array}{l}\text { Behavior } \\
\text { management } \\
\text { deficits }\end{array}$} & Increase antecedent strategies to reduce & Use demand fading, noncontingent reinforcement, and \\
\hline & & $\begin{array}{l}\text { differential reinforcement procedures. Increase Functional Communication } \\
\text { Training (FCT) to target mands for } \\
\text { attention, tangible, break, cessation, help, information, etc. }\end{array}$ \\
\hline & Modify the behavior plan to reduce & Ensure protocols are consumable for the caregiver. \\
\hline
\end{tabular}

complexity/

response effort.

Train the caregiver on implementation.

Technology deficits Train the caregiver in technology and troubleshooting.

Treatment concerns Have a meeting with caregiver to discuss concerns and collaboratively determine solutions.

No established Discuss telepractice rapport-building rapport procedures and provide examples.

Too much screen time Modify reinforcers to in-person reinforcers, not virtual/electronic.

Modify session structure in line with parent concerns when appropriate.

Cultural/role implications

Setting challenges
Engage collaboratively with the caregiver related to concerns and roles. Outline agreed-upon roles.

Work with the caregiver to identify a specific space for sessions.

Discuss setting modifications to reduce interruptions to sessions and family activities.
Outline specific roles for the behavior technician and caregiver related to protocol implementation.

Train with behavioral skills training.

Provide a task analysis for technology use and troubleshooting tips.

Model troubleshooting during caregiver training and record for later reference.

Train additional caregivers who may support (e.g., grandparent).

Use specific praise and feedback, and implement behavioral skills training.

Listen to parent concerns and suggest solutions that are clinically appropriate and that support caregiver values.

When possible, assign a behavior technician (BT) with established rapport. Consider supervisor overlapping with newly assigned BT during initial sessions.

Use scavenger hunts, crafts, pretend play, show-and-tell, dress-up, board games, painting, slime, etc.

Begin with short durations of telepractice and increase as the caregiver approves.

Build in breaks from screen time, snack time, movement breaks, etc.

Provide an outline/decision tree related to roles, responsibilities, and problem resolution.

Provide a workstation tip sheet.

Assign the learner tasks to develop and sustain a clean, organized workstation.

Provide the caregiver with instructions to support the learner's discrimination of work and play spaces for all family members. 

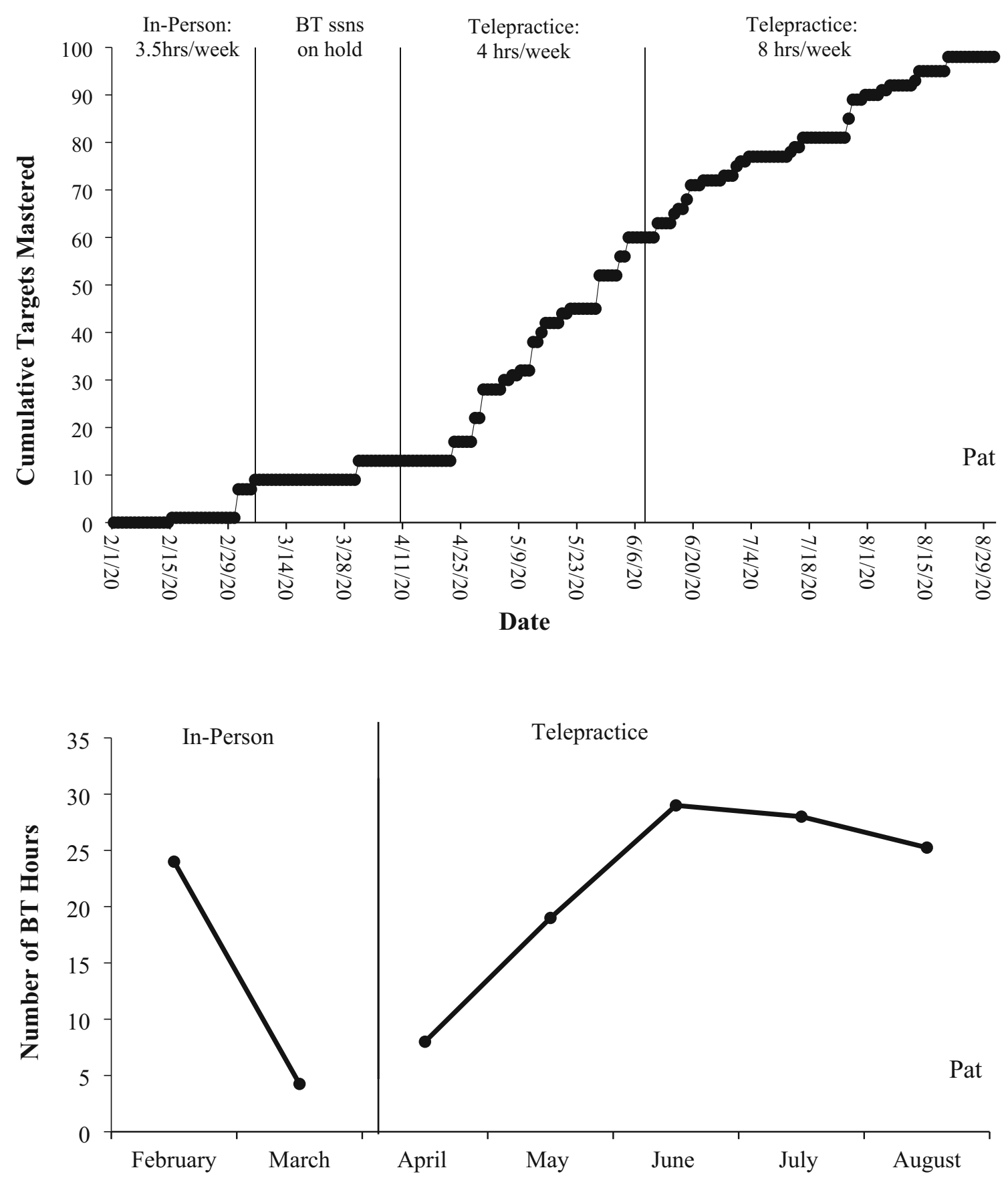

\section{Month of Service}

Fig. 2 Cumulative Targets Mastered and Behavior Technician (BT) Service Hours for Pat

involved shaping attending to visual and auditory stimuli, targeting generalization of mastered following instruction skills, and revising session structure and behavior reduction protocols. Because there was potential for learners with this profile to receive caregiver support, solutions also focused on training caregivers (e.g., prompting, restricting and delivering reinforcers, targeting generalization of prerequisite skills, and implementing behavior reduction strategies). This approach resulted in the successful implementation of BT telepractice despite learner deficits in prerequisite skills.

Case Study Larry was a 5-year-old boy diagnosed with ASD receiving an average of $8 \mathrm{hr}$ per week of insurance-funded BT-provided treatment prior to the SIP order. Larry spoke in full sentences and accessed his treatment with the use of natural environment teaching strategies during various play- 
based interactions. Larry had a history of engaging in tantrumming and physical aggression maintained by escape and attention; however, these behaviors had been successfully treated and were maintained at low rates during and outside of his treatment sessions. Larry's caregiver placed BT sessions on hold in late March, indicating concerns related to potential exposure to COVID-19. At that time, Larry's caregiver indicated interest in continuing to receive BCBA and BT intervention via telepractice; however, the funder of service had not yet approved BT-level telepractice. The BCBA continued with parent training and initiated the preparatory steps related to BT telepractice so services could be initiated immediately upon approval. The telehealth skills assessment indicated deficits in sustaining attending, waiting for attention, and following instructions. During telepractice probe sessions conducted by the BCBA, Larry engaged in elopement and tantrumming, which were determined to serve an escape function. BT telepractice was approved for funding in late April, and Larry's caregiver was willing to facilitate during sessions as needed. Larry began participating in $4 \mathrm{hr}$ of BT telepractice treatment weekly. Priority treatment targets included attending to the screen, sustaining engagement with the BT, waiting, following instructions, and manding. The BCBA increased the frequency of observations during initial BT telepractice sessions, during which support was provided via modeling and immediate performance feedback (using the chat function). Demand fading and functional communication training related to breaks were introduced to address elopement and tantrumming. Upon Larry demonstrating skill acquisition and successful treatment of his behavioral excesses (see Figure 3), caregiver participation was successfully faded to only a small portion of the session. Services were eventually increased to $8 \mathrm{hr}$ weekly of telepractice treatment (2-hr sessions, 4 days weekly). Rates of skill acquisition during telepractice were comparable to those during in-person treatment, and elopement and tantrumming were sustained at low rates.

\section{Profile 3: Learner With Prerequisite Skills and With Caregiver/}

Setting Barriers Similar to Profile 1, these learners demonstrated readiness skills, but caregiver and/or setting variables impacted implementation and were not readily remediated via caregiver collaboration. Additional measures were taken to address these variables to support learners and their caregivers in accessing intervention. Caregiver barriers were described as limited time (e.g., work and sibling demands); negative experiences with other online instructional formats, such as distance learning; caregiver deficits and lack of confidence in their ability to manage behavioral excesses or technology; and the reluctance of an alternative caregiver (e.g., grandparent) to follow practitioner recommendations or support the learner within sessions. Caregiver barriers most frequently reported by our team are outlined in Table 6 ; however, those observed as the most prevalent challenges to BT telepractice implementation were identified as time/participation constraints, motivation and efficacy concerns, and treatment concerns (e.g., too much screen time). Solutions were heavily focused on the following activities: (a) collaborative planning; (b) detailed and ongoing communication; (c) revision and clarification of schedules, roles/participation of the collaborative team, and session structure; (d) caregiver training; and (e) frequent sharing of learner successes.

Case Study Sidney was an 8-year-old boy with a diagnosis of ASD receiving $6 \mathrm{hr}$ of insurance-funded ABA intervention in his home prior to SIP restrictions. Sidney's parents immediately placed all in-person intervention on hold due to elderly grandparents living within the home. During initial collaborative planning meetings with the BCBA, caregivers reported that they were not interested in transitioning to telepractice, citing being overwhelmed with work responsibilities. As recommended, the BCBA maintained weekly check-ins with the caregivers to explore any shifts in barriers to service delivery, propose solutions, and offer support. After approximately 6 weeks without BT-level intervention services, caregivers agreed to a learner assessment of telepractice readiness skills. The assessment results indicated that Sidney demonstrated the prerequisite skills for BT telepractice. The BCBA shared the results and asked the caregivers if she could conduct a 30-min telepractice probe. Caregivers agreed to a short session during which Sidney demonstrated progress toward targeted treatment goals. Subsequently, the BCBA shared the data from the session, as well as anecdotal reports of positive statements made by Sidney related to his experience. Caregivers agreed to reinitiate intervention via BT telepractice, and Sidney resumed receipt of $6 \mathrm{hr}$ of intervention weekly after several weeks of no access to intervention and $0 \mathrm{hr}$ of service provided. Sidney acquired skills during BT telepractice during the 1st month of sessions (see Fig. 4).

Profile 4: Learner Without Prerequisite Skills and With Caregiver/Setting Barriers Learners meeting this profile were found to be the most challenging for whom to remediate access issues via BT telepractice. These learners required more extensive skill development to fully participate in telepractice sessions. In these cases, the level of skill deficit required higher rates of caregiver support and participation in sessions, and the caregivers were less willing to initiate sessions. Learners categorized under this profile were reported to require structured support and potentially in-person facilitation related to prompting and implementation of behavior protocols. Caregivers stated concerns related to the learners' deficits, the inability to provide their hypothesized required levels of assistance due to conflicting demands (e.g., work), and worries that access to virtual reinforcers during sessions may reduce the caregivers' ability to use "screen access" to occupy 

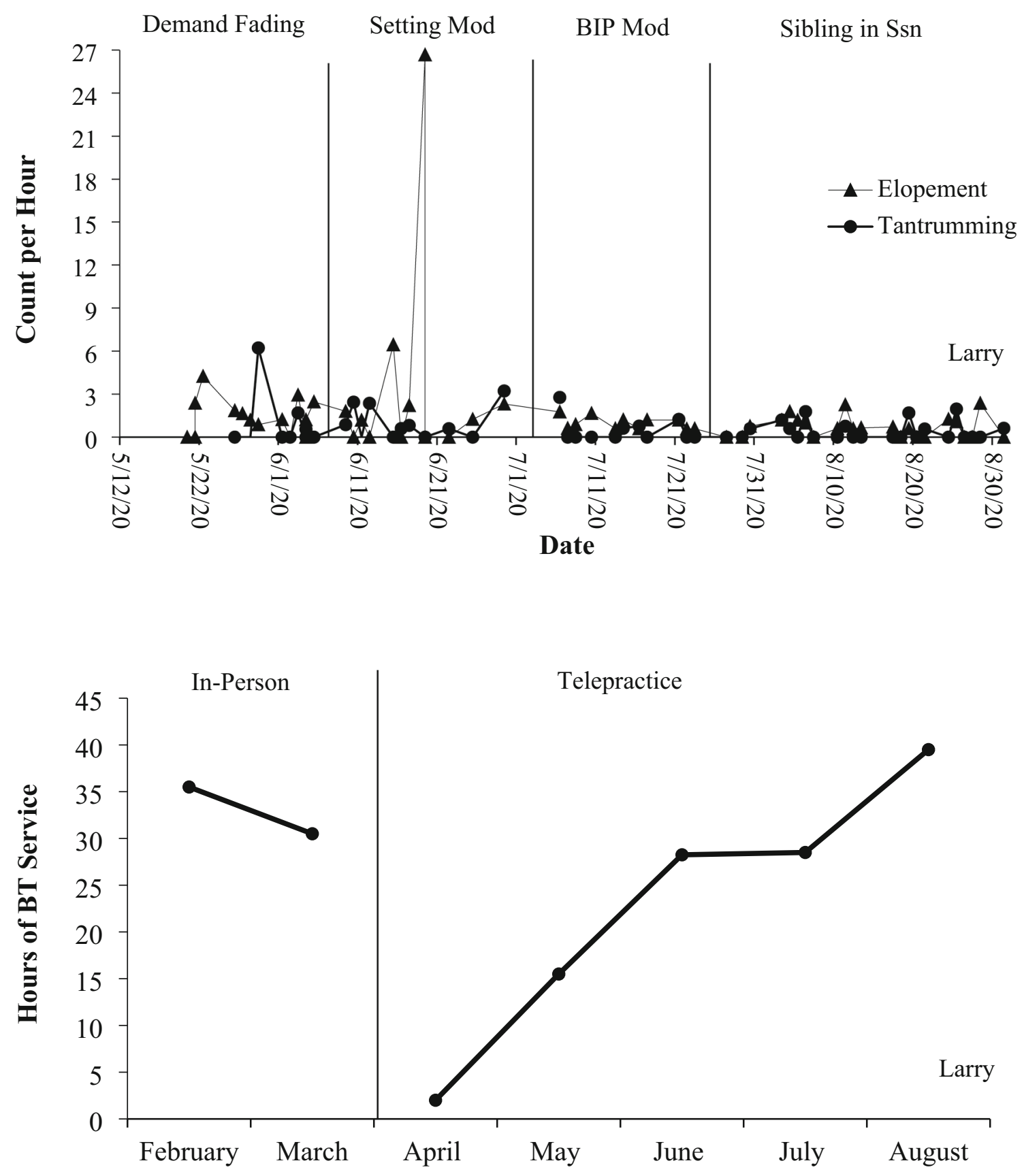

Month of Service

Fig 3 Undesirable behavior rates and Behavior Technician (BT) service hours for Larry. Note. BIP = Behavior Intervention Plan; Mod = Modification

the learner outside of sessions so caregivers could complete required work and household duties. The intersection of both learner skill deficits and caregiver/setting barriers most often required the supervising clinical team to shape the learner's prerequisite skills with no or minimal caregiver facilitation. Solutions frequently involved BCBAs and/or midlevel supervisors conducting direct telepractice sessions with learners to target attending and instruction-following skills, as well as increases in antecedent strategies to reduce the probability of behavioral excesses and the need for caregiver support during sessions.

Case Studies Steven was a 4-year-old boy diagnosed with ASD receiving an average of $6 \mathrm{hr}$ per week of insurancefunded BT-provided treatment (due to his limited availability) prior to the SIP order. Steven spoke in short sentences and accessed his treatment with the use of natural environment teaching strategies during various play-based interactions. 


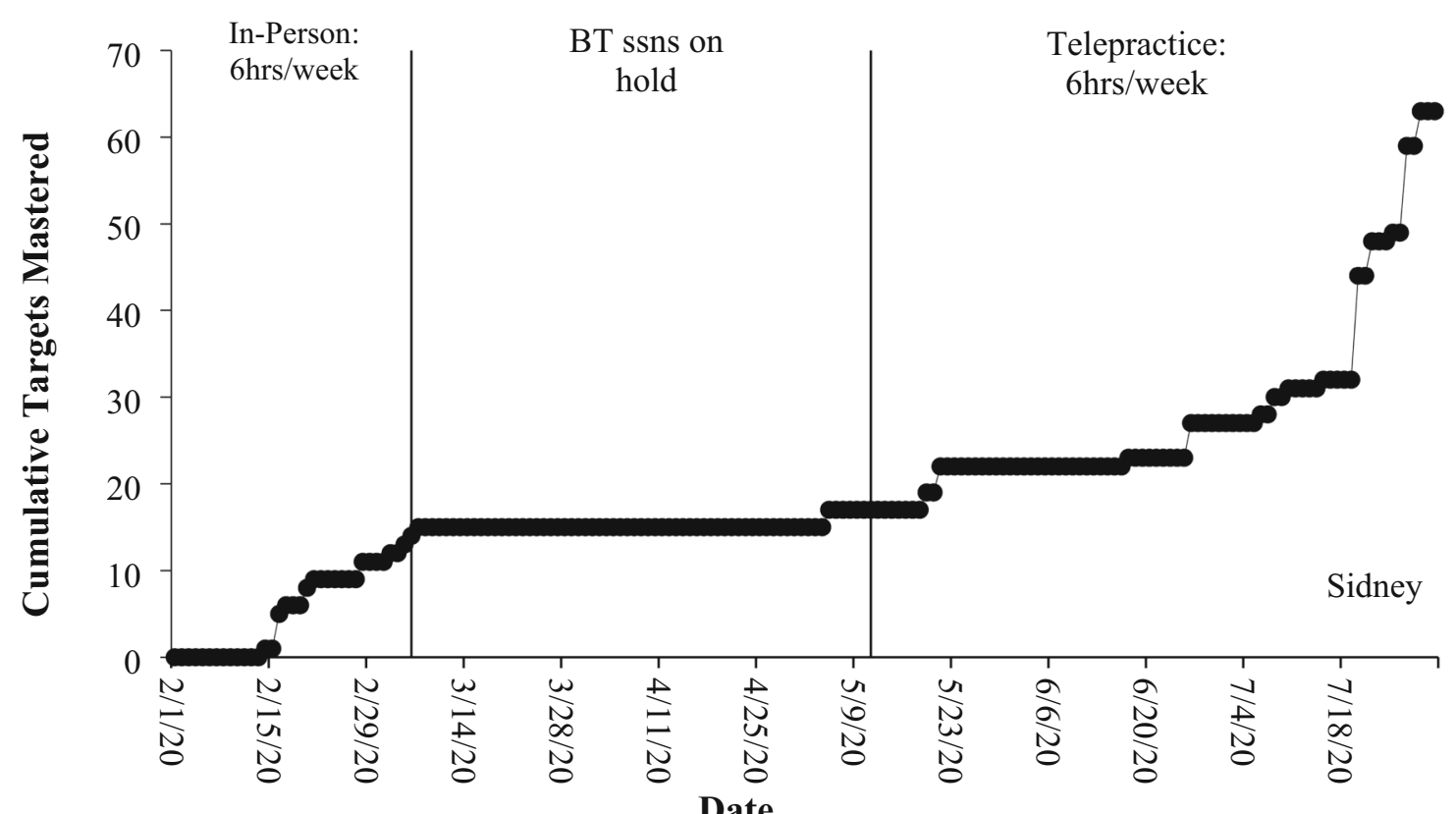

Date

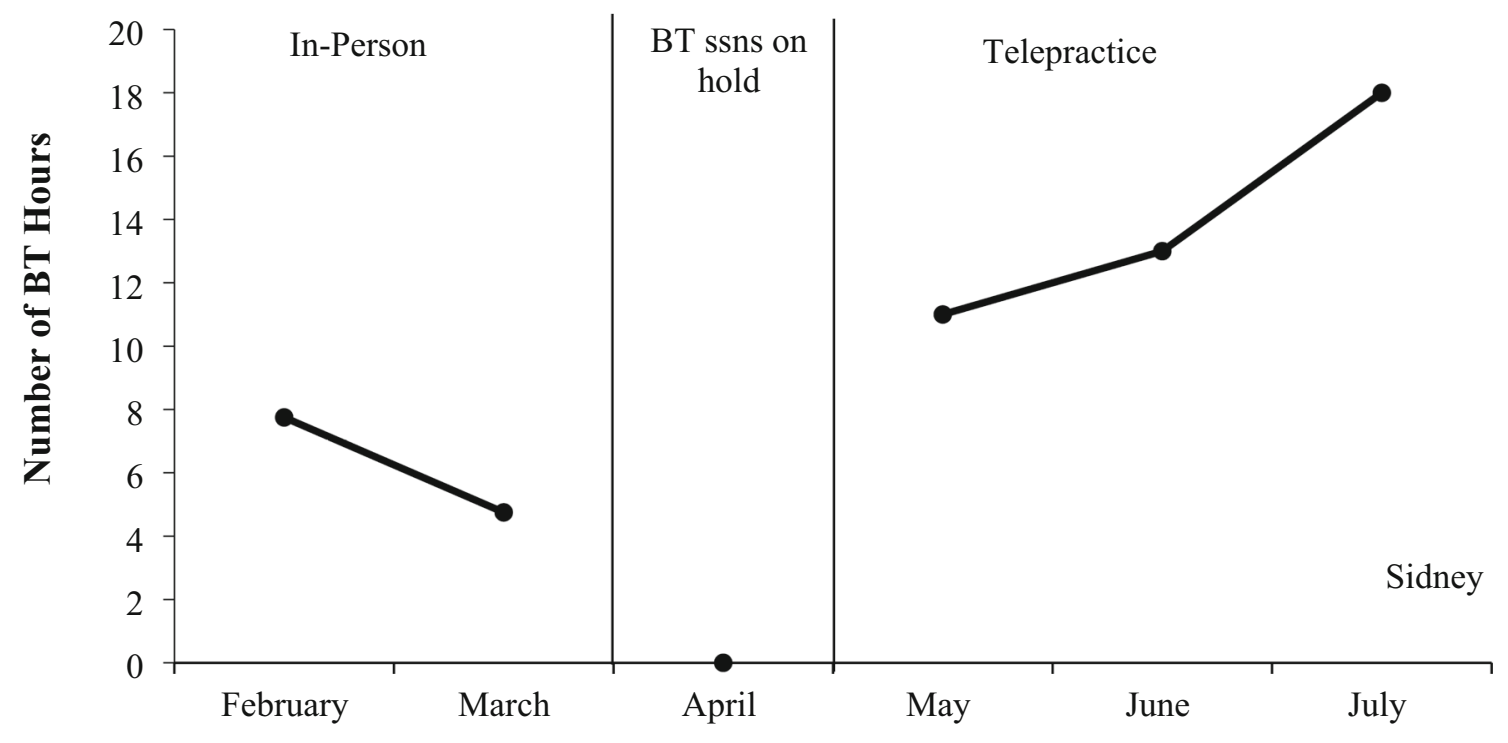

Month of Service

Fig. 4 Cumulative targets mastered and Behavior Technician (BT) service hours for Sidney

He moved frequently throughout the home and engaged in tangible- and escape-maintained tantrumming. Citing being uncomfortable with having the BTs inside the home during the SIP order, Steven's caregiver immediately placed sessions on hold. Initially, Steven's caregiver indicated that telepractice did not seem to be a possibility for Steven, given his skill deficits, his challenging behavior, and the caregiver's difficulties providing the level of support that may be needed. The BCBA and caregiver met to openly discuss the potential barriers and identify agreed-upon solutions. Barriers related to Steven's skill set centered around deficits in attending, engagement, and following instructions, as well as behavioral excesses that his mother did not think she could support as needed. Reported caregiver and environmental barriers included time and participation constraints (e.g., his mother attempting to work from home and manage Steven and his siblings, who may also need support with telepractice sessions or school), technology deficits (e.g., inconsistent internet 
connectivity, limited access to the number of devices needed for each child at home), treatment concerns (e.g., too much screen time), and an overall lack of motivation for telepractice sessions. During meetings with the caregiver, these barriers were addressed and prioritized (see Tables 5 and 6 for more detail). With caregiver consent, the telepractice skills assessment and probe session were conducted, and the team decided that telepractice was a viable option with modifications to reduce the amount of parental involvement required. Priority treatment targets included attending to the screen, engaging with the BT for extended durations, complying, and speaking at an audible volume. Modifications to the session schedule occurred, allowing Steven and a sibling to have sessions at the same time, maximizing the available electronics while minimizing time requirements for the caregiver. These modifications, along with discussions regarding the clinical recommendations and potential change in outcome trajectory, resulted in the caregiver agreeing to telepractice treatment sessions. These began with a supervisor conducting telepractice sessions to establish the foundational telepractice skills and to systematically increase session duration. BT-level treatment was then provided. Frequent modifications to session structure, reinforcement protocols, and behavior protocols occurred as needed to address shifting barriers. Early in the telepractice treatment, frequent check-ins occurred with Steven's caregiver to assess her comfort level with telepractice sessions, discuss any needed modifications, and provide updates regarding progress. Services were eventually increased to $5 \mathrm{hr}$ per week of telepractice treatment (1-hr sessions, 5 days a week). His rates of skill acquisition during telepractice were comparable to when he received $5 \mathrm{hr}$ of in-person treatment (Fig. 5).

Not all learners meeting the criteria for Profile 4 will necessarily experience a decrease in BT-level service hours, as risks may be safely mitigated such that in-person intervention can continue. However, proactively meeting these criteria increases the probability that if conditions changed, and in-person intervention was no longer a viable option, the negative impact on needed intervention could be minimized. If one takes the position that all learners in the presence of ever-evolving conditions associated with the pandemic may require telepractice to sustain access to services, then an investment in establishing these prerequisite skills seems worthwhile, even while a learner is still accessing inperson intervention.

This approach was implemented with Phillip. Phillip was a 7-year-old boy diagnosed with ASD receiving $10 \mathrm{hr}$ of inperson, insurance-funded BT intervention prior to school closures. Following school closure, he was available to access his full treatment authorization of $30 \mathrm{hr}$ weekly. Phillip lacked telepractice readiness skills. He had a history of behavioral excesses, including property destruction, physical aggression, and tantrumming. Phillip's caregiver was motivated to increase and sustain in-person intervention given reported barriers to telepractice, including caring for and managing distance learning for Phillip's three siblings. Phillip's caregiver was also reluctant to explore telepractice due to reported difficulties with managing Phillip's undesirable behaviors and a poor experience with his distance-learning program provided by the school district. In later collaborative planning meetings, the caregiver and BCBA agreed to increase in-person treatment to match recommended service rates, while also introducing telepractice readiness skill development as a preparatory measure should access to in-person treatment be reduced given pandemic-related variables.

Skills introduced as components of this preparatory program included attending to the screen, following instructions, manding for breaks from telepractice, and generalizing skills previously acquired in person to telepractice conditions. These skills were addressed during a 30- to 60-min weekly telepractice session (with increasing durations), wherein Phillip was provided with in-person BT support, whereas the midlevel supervisor served as the clinician delivering the telepractice intervention. More specifically, instruction was delivered remotely by the supervisor via a virtual platform, whereas the BT present provided any required in-person prompting and reinforcement. The structure of these telepractice sessions transitioned over time to a format more closely approximating a telepractice session without in-person BT support (i.e., the remote clinician was able to more successfully prompt and reinforce responding). Figure 6 depicts the acquisition of these skills during the telepractice readiness portions of treatment sessions. Phillip continued acquiring additional telepractice readiness skills and generalizing previously mastered skills to that mode of treatment. Moreover, caregiver report and direct observation suggest that some of these skills generalized to his distance-learning educational program provided by his local district, in the absence of BT support. These steps toward preparing Phillip for telepractice required thoughtful development, collaboration, and implementation that have better prepared him to access intervention as needed.

The categorization and problem resoluation process for Profile 4 learners was also implemented at the group level, specifically within a preschool classroom. This is included as an initial demonstration of a larger scale application in a context with barriers involving multiple caregivers/ parties (parents, administrators, and funders). The learners within the collaborative preschool met the criteria for Profile 4. Our organization initiated support for a collaborative special day preschool program in late January 2020, and schools were later closed to mitigate the spread of COVID-19. The preschool served 10 students, ranging in age from 3 to 6 years, who qualified for special education services under a variety of educational diagnoses, including speech and language impairment, intellectual disability, and ASD. Prior to school closure, 

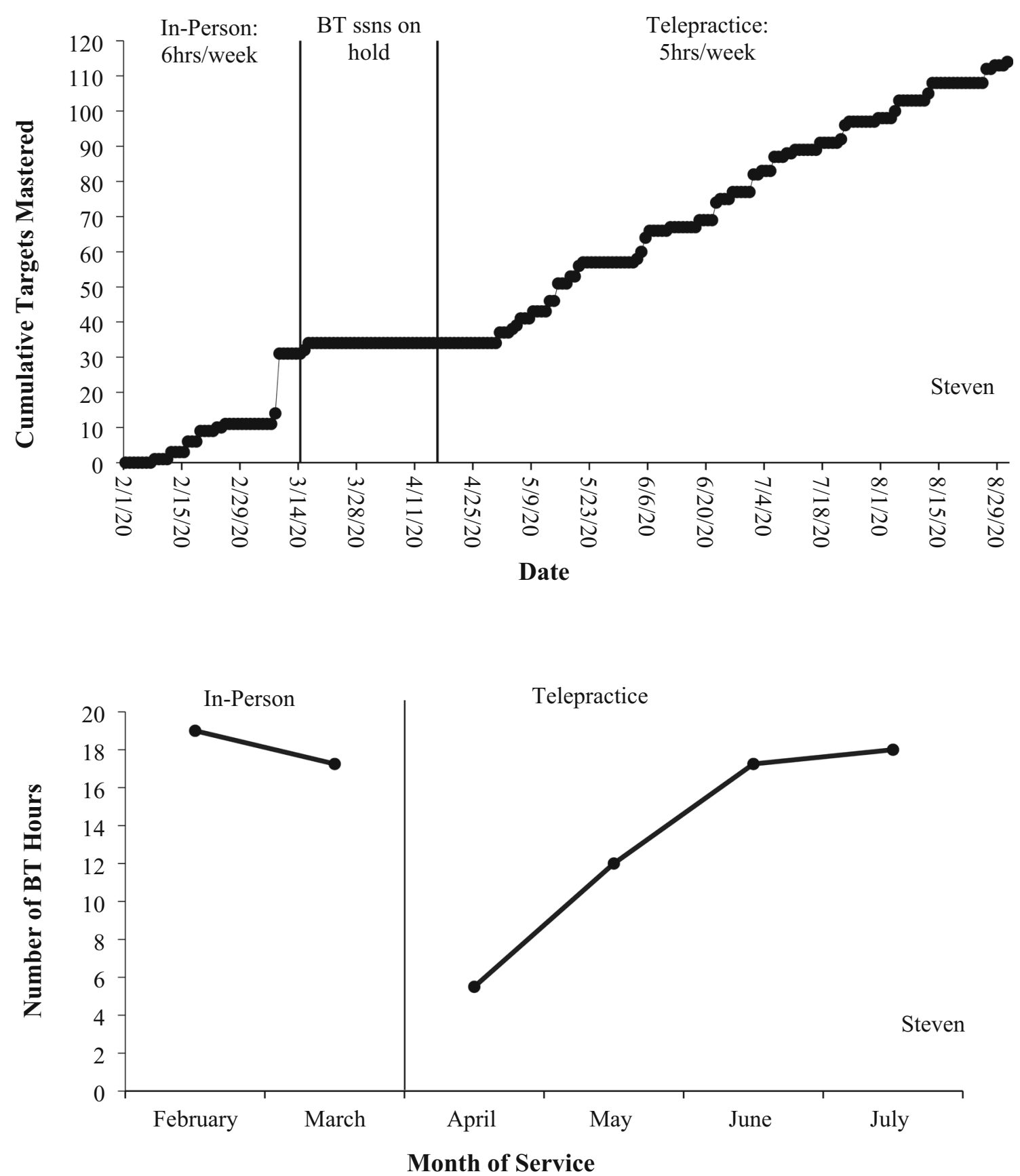

Fig. 5 Cumulative targets mastered and Behavior Technician (BT) service hours for Steven

the preschool students participated in a 20 -hr weekly oncampus program. Our organization provided the program and its students with full school day support 5 days a week via a midlevel supervisor and BT. In addition, BCBA supervision focused on program development, staff training, and individual learner supports (e.g., behavior intervention plan development) up to $8 \mathrm{hr}$ weekly.

These young learners all lacked telepractice readiness skills. Initially, the learners' deficits made it challenging for the collaborative team (i.e., teachers, special service providers, administrators, and caregivers) to envision how the learners could be effectively served under a telepractice model. These combined barriers resulted in the learners being served through the provision of instructional packets to be completed by the students with their caregivers. This format of instruction (no ABA intervention and instructional packets only) continued for a period of approximately 6 weeks.

During this period, advocacy and collaboration with school district administrators resulted in the initiation of direct service via telepractice. The disruption in service 

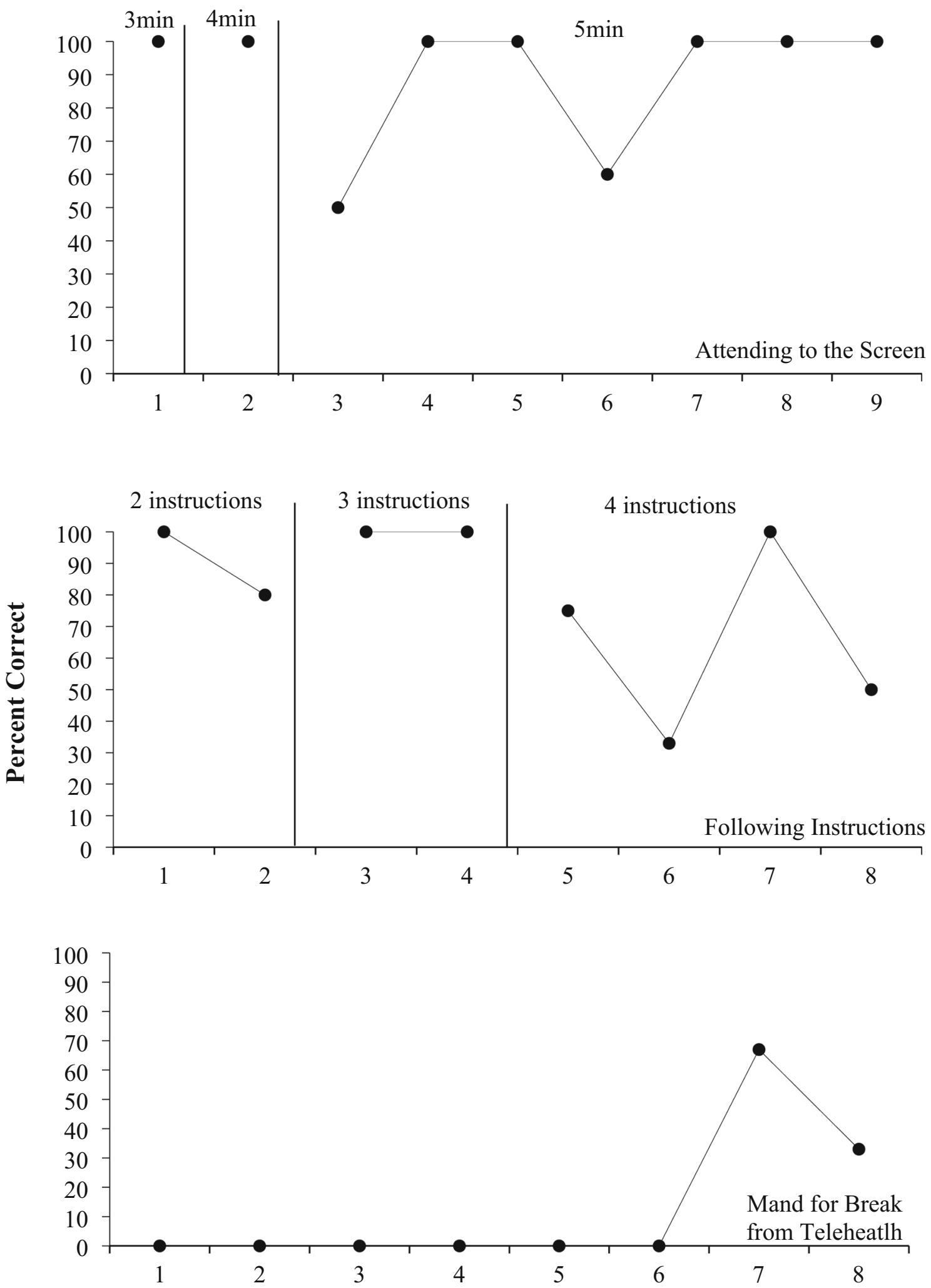

Session

Fig. 6 Acquisition of telepractice prerequisite skills for Phillip

and the coordination of collaborative team members were challenges for the remaining weeks of the school year, as reflected in learner attendance in direct telepractice sessions; however, a number of steps were taken to increase 
access during the offered summer program. The specific steps included surveying and conducting open-ended interviews with caregivers to examine their satisfaction with the models of distance support provided during the spring, expanding the number of small- and large-group synchronous instruction provided daily to learners led by midlevel supervisors and BTs, conducting related caregiver training (e.g., preparing workstations, establishing a routine, and preparing for distance-learning sessions), meeting with each caregiver individually to review the model for summer program instruction, and including individualized BT telepractice sessions with each learner two to three times weekly. Levels of service increased from $0 \mathrm{hr}$ of service during the initial weeks of school closure to $3 \mathrm{hr}$ of direct synchronous instruction delivered in small groups, and then to $10 \mathrm{hr}$ of direct synchronous instruction during the summer program delivered in small-group and individualized formats, as well as $10 \mathrm{hr}$ of asynchronous, videobased instruction (i.e., $20 \mathrm{hr}$ of instruction) created and conducted by our team. In summary, learners in this preschool program transitioned from no direct instruction and a packet-only format to $20 \mathrm{hr}$ of weekly instruction involving both synchronous and asynchronous instruction. In addition, data collected throughout all phases revealed decreased cancellations and increased participation as the level of service hours increased.

\section{Results and Discussion}

Our organization serves approximately 270 learners. Over the course of a 6-month period, more than 82 of these learners had experienced some level of BT telepractice. Figure 7 shows the number of learners across funding sources (school district and insurance funding) who received BT telepractice hours from February through August 2020. Learners supported by the NPA ranged in age from 3 to 18 years and were served under various educational placements, from special day classrooms, to fully inclusive general education placements. These learners received $100 \%$ of their ABA services via telepractice. The majority of learners in our NPS continued to receive inperson BT support in their homes, and their teachers and special service providers conducted sessions via telepractice. Some learners, however, required BT telepractice in order to access this support due to comprised family members or other environmental variables. Data collected indicate that $34 \%$ of the total number of BT-level insurance-funded service hours were delivered via telepractice. For the majority of these learners, these hours were delivered via telepractice because in-person treatment was not an option. In the absence of BT telepractice, access to service for these learners would be reduced or absent.

Figure 8 shows the cumulative number of BT telepractice service hours provided by our organization across funding sources starting in February. School closures in mid-March eliminated access to ABA intervention for all learners served by our NPA. Through advocacy and collaborative efforts, we were able to secure and initiate BT telepractice for all learners served by the NPA, as well as those served by the NPS whose caregivers selected telepractice service delivery over in-person BT support. Prior to March 30, 2020, our organization had provided $0 \mathrm{hr}$ of telepractice. In the final 2 days of March, 218 BT telepractice hours were provided to learners via the NPA or NPS. BT-level telepractice was not approved by any funders of learners receiving our insurancebased services until early April 2020. We observed an $88 \%$
Fig. 7 Insurance- and school district-funded learners receiving Behavior Technician (BT) telepractice

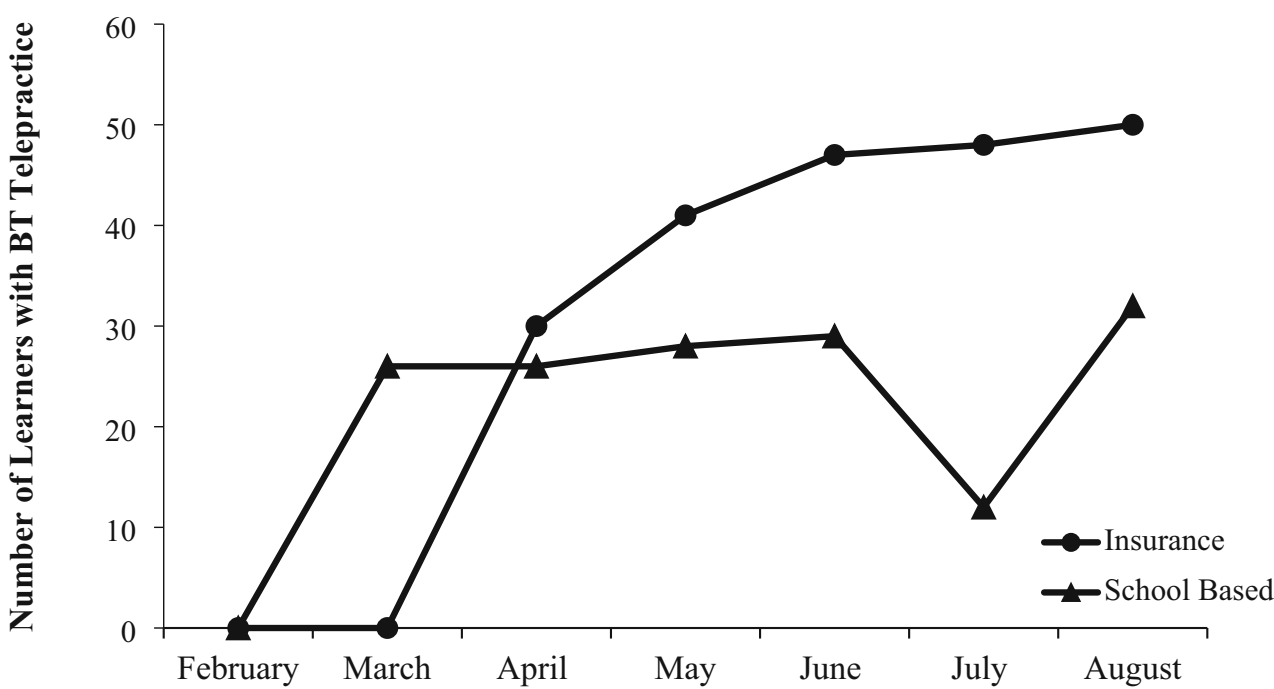

Month of Service 


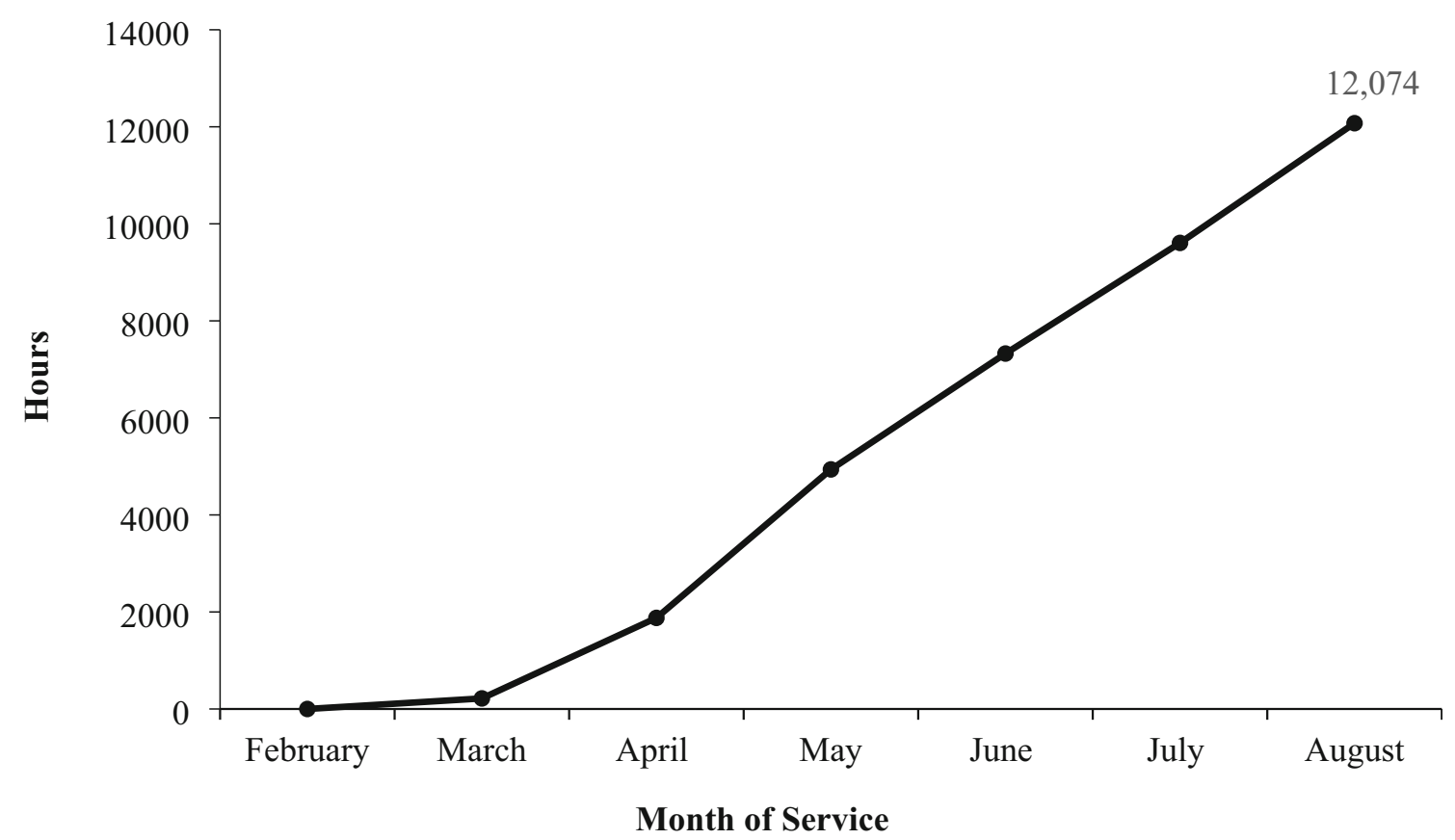

Fig. 8 Cumulative number of Behavior Technician (BT) telepractice hours

increase in cumulative BT telepractice hours from March (218 hr) to April $(1,877 \mathrm{hr})$. BT telepractice hours continued to increase across consecutive months, with drastic increases evident from March through June 2020. Slight decreases in monthly BT telepractice hours were evident in the months of July and August due to periods of no service as a result of summer breaks for our NPA and NPS learners. During these months, overall increases in BT telepractice hours for learners receiving insurance-funded services were still evident. Our organization provided a cumulative total of 12,704 hr of BT telepractice between March and August 2020.

\section{Social Validity}

Our efforts to increase access to recommended ABA intervention have resulted in favorable outcomes for many of our learners receiving telepractice services at the BT level, as indicated by skill acquisition and behavior reduction data. Still, the social acceptance of this relatively novel approach to intervention must be evaluated. Social validity surveys were sent to the caregivers of all of our learners receiving insurance-funded telepractice services at the BT level, asking for their impressions regarding the telepractice service their child had received. Of the respondents, $63 \%$ of caregivers reported the most preferred feature of telepractice was increased safety during the pandemic. Other common responses included receiving support with challenging behavior and observing and learning from the BT. Seventy-three percent of respondents indicated that they were satisfied with the mode of service delivery, as well as with the rate and duration of treatment in this modality. More than $90 \%$ of respondents indicated that they preferred more or the same amount of telepractice intervention.

Though the majority of caregivers receiving insurancefunded BT telepractice services indicated overall satisfaction with the learner-specific treatment goals, procedures, and outcomes, there were still several who reported an uncertainty with respect to their satisfaction with this mode of treatment. These data, taken together with learner data and ongoing efforts toward improving treatment outcomes and social validity, guide us to continually evolve our clinical practices, especially within the realm of telepractice. Telepractice at the level of the BT is new and less familiar to learners and their caregivers. We plan to use our growing knowledge and database to support making meaningful improvements toward the social validity of this form of intervention. Continued collaboration with caregivers and team members will be essential in this regard.

\section{Conclusion}

The pandemic has presented many difficulties for our learners, their families, our team, and our external collaborative partners. Here, we addressed our approach and offered potential solutions to support sustained access to ABA intervention, as we recognize that this challenge extends well beyond learners in our immediate reach. We acknowledge that sustained access to critical services such as ABA is only one plight among the wide-ranging and deleterious impacts of COVID-19. There were obstacles as we moved through the steps shared here to prepare learners for immediate or impending 
transitions to telepractice, worked toward agreements with funding agencies to implement ABA intervention remotely as authorized, and supported our internal team with problem resolution. We acknowledge the prevalence of barriers to access that many are facing in the context of novel and precarious conditions. We share our model, materials, experiences, preliminary data, and components related to problem resolution in an effort to assist practitioners, particularly those who may have less control over their clinical setting (e.g., consultants, BCBAs employed by a school district) or those who are not supported by a team of seasoned BCBAs. This information is shared in the hope that it can help practitioners overcome obstacles that may be reducing or entirely preventing access to ABA intervention for learners. Practitioners may use similar methods coupled with systematic data collection and modifications to the steps outlined here if they are curbed in their efforts to create access for their learners.

The model presented and steps taken to sustain access to ABA intervention and supports for our learners require continued analysis to evaluate the effectiveness of the model's components and their potential contributions to outcomes at the level of individual learners and sustained service delivery at the organizational level. Furthermore, and a limitation of the work presented here, there is no experimental evidence that the model, practices, or tools are more effective than alternatives that have been implemented by other behavior analysts. Another potential limitation relates to the applicability of our experiences with telepractice to those of other providers. Other providers may have very different practice experiences, including serving different populations, operating under a more unitary funding source, or practicing in other states. With respect to the final variable, our organization is based in California, wherein state law mandates equity in legal rights and responsibilities for persons with developmental disabilities (Lanterman Developmental Disabilities Services Act, 2020). Therefore, the levels of support from regulatory agencies such as school districts may be greater than those found in other states. Another critical limitation with the models and steps outlined here is that they necessitate access to technology, equipment, and the internet. Many learners and their families are lacking these fundamental resources. School districts serving learners attempted to address such accessibility issues through the provision of Chromebooks and air cards for internet connectivity, yet disparities and difficulties may still persist. It is recommended that $\mathrm{ABA}$ providers maintain an awareness of learners' needs for critical resources and consider what steps they may take to support access and equity for all learners.

The collective recent dissemination efforts (e.g., practice guidelines, journal publications, and webinars by BCBAs and supporting associations) are demonstrations of valuesbased actions to ensure continued services for learners under conditions that necessitate fundamental changes in service delivery. Behavior analysts need to sustain and widen these efforts, making certain that experiences and data are shared to support the further refinement of these practices, as well as the ability to advocate for their implementation and funding. It is also important to recognize that the work behavior analysts are doing, and the systematic analysis of that work, is relevant to practitioners outside of behavior analysis who are delivering support services. Sharing these early findings with individuals working within other service domains, such as education and health care, may help more learners access critical supports that have been impacted by or reduced as a result of the pandemic. Data and a commitment to its dissemination can assist in eliminating barriers for learners. Collecting data and disseminating them might also eliminate barriers existing between behavior analysts working across agencies, populations, and funding sources and those working outside of applied behavior analysis. This dissemination should focus on sharing models, experiences, and outcomes as a means to support one another.

Supplementary Information The online version contains supplementary material available at https://doi.org/10.1007/s40617-020-00550-8.

Funding The authors did not receive specific funding for this study.

Availability of Data and Material The data sets generated and analyzed during the current study are available from the corresponding author upon reasonable request.

Code Availability Not applicable; no code was used for this study.

\section{Declarations}

Consent to Participate Informed consent was obtained in order to conduct a retrospective analysis of treatment data.

Consent for Publication Informed consent was obtained in order to publish a retrospective analysis of treatment data.

Conflicts of Interest The authors declare that they have no conflict of interest.

\section{References}

Boisvert, M., Lang, R., Andrianopoulos, M., \& Boscardin, M. L. (2010). Telepractice in the assessment and treatment of individuals with autism spectrum disorders: A systematic review. Developmental Neurorehabilitation, 13(6), 423-432. https://doi.org/10.3109/ 17518423.2010 .499889

California Department of Developmental Services. Lanterman Developmental Disabilities Services Act and related laws. 
Retrieved November 15 2020, from https://www.dds.ca.gov/wpcontent/uploads/2020/03/DDS_LantermanServicesAct_2020.pdf

Colombo, R. A., Wallace, M., \& Taylor, R. (2020). An essential service decisions model for applied behavior analytic providers during crisis. Behavior Analysis in Practice, 13, 306-311. https://doi.org/10. 31234/osf.io/te8ha

Ferguson, J., Craig, E. A., \& Dounavi, K. (2019). Telehealth as a model for providing behaviour analytic interventions to individuals with autism spectrum disorder: A systematic review. Journal of Autism and Developmental Disorders, 49(2), 582-616. https://doi.org/10. 1007/s10803-018-3724-5.

Frederick, J. K., Raabe, G. R., Rogers, V. R., \& Pizzica, J. (2020). Advocacy, collaboration, and intervention: A model of distance special education support services amid COVID-19. Behavior Analysis in Practice, 13, 748-756. https://doi.org/10.1007/s40617-02000476-1.

Hayes, S. C., Barlow, D. H., \& Nelson-Gray, R. O. (1999). The scientist practitioner: Research and accountability in the age of managed care (2nd ed.). Allyn \& Bacon.

Healy, O., \& Sinéad, L. (2013). Early intensive behavioural intervention in autism spectrum disorders. In M. Fitzgerald (Ed.), Recent advances in autism spectrum disorders (pp. 567-597). IntechOpen. https://doi.org/10.5772/54274.

LeBlanc, L. A., Lazo-Pearson, J. F., Pollard, J. S., \& Unumb, L. S. (2020). The role of compassion and ethics in decision making regarding access to applied behavior analysis services during the COVID-19 crisis: A response to Cox, Plavnick, and Brodhead. Behavior Analysis in Practice, 13, 604-608. https://doi.org/10. 1007/s40617-020-00446-7

Lindgren, S., Wacker, D., Suess, A., Schieltz, K., Petzel, K., Kopelman, T., Lee, J., Romani, P., \& Waldron, D. (2016). Telehealth and autism: Treating challenging behavior at lower cost. Pediatrics, 137(Suppl. 2), S167-S175. https://doi.org/10.1542/peds.201528510.

Rodriguez, K. A. (2020). Maintaining treatment integrity in the face of crisis: A treatment selection model for transitioning direct ABA services to telehealth. Behavior Analysis in Practice, 13, 291-298. https://doi.org/10.1007/s40617-020-00429-8.
Romani, P. W., \& Schieltz, K. M. (2017). Ethical considerations when delivering behavior analytic services for problem behavior via telehealth. Behavior Analysis: Research and. Practice, 17(4), 312324. https://doi.org/10.1037/bar0000074.

Schieltz, K. M., \& Wacker, D. (2020). Functional assessment and function-based treatment delivered via telehealth: A brief summary. Journal of Applied Behavior Analysis, 53, 1242-1258.

Scudellari, M. (2020). How the pandemic might play out in 2021 and beyond. Nature. Retrieved on September 15 2020. https://www. nature.com/articles/d41586-020-02278-5

Simacek, J., Dimian, A. F., \& McComas, J. J. (2017). Communication intervention for young children with severe neurodevelopmental disabilities via telehealth. Journal of Autism and Developmental Disorders, 47, 744-767. https://doi.org/10.1007/s10803-016-3006$\mathrm{z}$.

The Council of Autism Service Providers. (n.d.). Telehealth and crisis management guidelines. Retrieved from https://casproviders.org/ telehealth-and-crisis-management-guidelines/

Tomlinson, S. R., Gore, N., \& McGill, P. (2018). Training individuals to implement applied behavior analytic procedures via telehealth: A systematic review of the literature. Journal of Behavioral Education, 27, 172-222.

United Nations. (2020). Policy brief: Education during Covid-19 and beyond. https://www.un.org/development/desa/dspd/wp-content/ uploads/sites/22/2020/08/sg_policy_brief_covid-19_and_ education august 2020.pdf

Wacker, D. P., Leeb, J. F., Padilla Dalmaub, C., Kopelman, T., Lindegren, S., Kuhleb, K. E., Dyson, S., Schieltzh, K. M., \& Waldrona, D. B. (2013). Conducting functional communication training via telehealth to reduce the problem behavior of young children with autism. Journal of Developmental and Physical Disabilities, 25(1), 35-48.

Publisher's Note Springer Nature remains neutral with regard to jurisdictional claims in published maps and institutional affiliations. 